\title{
Time-Domain Reconstruction Algorithms and Numerical Simulations for Thermoacoustic Tomography in Various Geometries
}

\author{
Minghua Xu, Yuan Xu, and Lihong V. Wang*, Senior Member, IEEE
}

\begin{abstract}
In this paper, we present time-domain reconstruction algorithms for the thermoacoustic imaging of biological tissues. The algorithm for a spherical measurement configuration has recently been reported in another paper. Here, we extend the reconstruction algorithms to planar and cylindrical measurement configurations. First, we generalize the rigorous reconstruction formulas by employing Green's function technique. Then, in order to detect small (compared with the measurement geometry) but deeply buried objects, we can simplify the formulas when two practical conditions exist: 1) that the high-frequency components of the thermoacoustic signals contribute more to the spatial resolution than the low-frequency ones, and 2) that the detecting distances between the thermoacoustic sources and the detecting transducers are much greater than the wavelengths of the high-frequency thermoacoustic signals (i.e., those that are useful for imaging). The simplified formulas are computed with temporal back projections and coherent summations over spherical surfaces using certain spatial weighting factors. We refer to these reconstruction formulas as modified back projections. Numerical results are given to illustrate the validity of these algorithms.
\end{abstract}

Index Terms-Algorithm, geometry, imaging, photoacoustics, reconstruction, thermoacoustics, time-domain, tomography.

\section{INTRODUCTION}

$\mathbf{R}$ ECENT research has suggested that thermoacoustic tomography using either pulsed radio-frequency (RF) [1]-[8] or pulsed laser [9]-[12] can be a powerful imaging technology with good spatial resolution. Within this technique, when a pulsed electromagnetic irradiation is absorbed by a tissue, the heating and subsequent expansion of the tissue give rise to an instantaneous acoustic stress or pressure distribution inside the tissue. Directly following the pulse irradiation, the induced pressure distribution prompts acoustic wave propagation toward the surface of the tissue with various time delays. Ultrasound detectors are placed around the tissue to record the outgoing acoustic waves. These detected acoustic waves can be

Manuscript received September 24, 2001; revised February 8, 2003. This work was sponsored in part by the U.S. Army Medical Research and Materiel Command under Grant DAMD17-00-1-0455, in part by the National Institutes of Health (NIH) under Grant R01 CA71980, in part by the National Science Foundation (NSF) under Grant BES-9734491, and in part by the Texas Higher Education Coordinating Board under Grant ARP 000512-0123-1999. Asterisk indicates corresponding author.

M. Xu and Y. Xu are are with the Optical Imaging Laboratory, Department of Biomedical Engineering, Texas A\&M University, College Station, TX 77843-3120 USA.

*L. V. Wang is with the Optical Imaging Laboratory, Department of Biomedical Engineering, Texas A\&M University, 3120 TAMU, College Station, TX 77843-3120 USA(e-mail: LWang@tamu.edu; URL: http://oilab.tamu.edu). Digital Object Identifier 10.1109/TBME.2003.81608 used to inversely compute the distribution of the initial acoustic pressure or electromagnetic absorption, which is related to the properties of the tissue.

In fact, electromagnetic fields in the RF range of 300 to 3000 $\mathrm{MHz}$ are the most useful in the study of soft tissues sized in centimeters. The RF penetration depth at this frequency range varies depending on the tissue properties and the RF frequency [3], [13], [14]. For example, the penetration depths for muscle and fat are about 1.2 and $9 \mathrm{~cm}$ at $3 \mathrm{GHz}$, respectively, and about 4 and $30 \mathrm{~cm}$ at $300 \mathrm{MHz}$, respectively; most other soft tissues have penetration depths that fall between these values. In addition, in this frequency range, there is very little scattering by the tissues [13].

In a typical application of thermoacoustic imaging using RF, a short-pulsed RF field illuminates the tissue. The most investigated and documented effect of RF power on biological tissues is the transformation of energy entering the tissues into increased kinetic energy in the absorbing molecules, thereby producing a general heating in the medium [13]. The heating results from both ionic conduction and vibration of the dipole molecules of water and proteins [13]. The energy absorbed by the tissue produces a temperature rise that is dependent on the cooling mechanism of tissue [13]. Human exposure to RF power must be limited for safety reasons, and within the mandated safety limits, the temperature rise per short pulse (such as $1 \mu \mathrm{s}$ ) in soft tissue is very small (on the order of milli-degrees) [6].

Nevertheless, this small temperature rise causes linear expansion of the tissue. The heating and expansion are greatest in those regions of the tissue that absorb the most RF power. Therefore, a distribution of acoustic pressure or stress inside the tissue is induced immediately during the short RF-pulse irradiation period due to heterogeneities of the RF energy deposition and the Grüneisen parameter inside the inhomogeneous tissue. Thermal expansion due to energy deposition is commonly referred to as the thermoelastic effect [15]. The generated acoustic pressure is on the order of mBar [6]. Such a small value does not cause tissue damage.

Subsequently, after the RF-pulse irradiation, the acoustic stresses inside the tissue relax. They act as instantaneous acoustic sources inside the tissue, which promote acoustic wave propagation. These acoustic waves contain acoustic frequencies ranging from very low frequencies to high frequencies that approximate the reciprocal of the RF pulse duration. The acoustic detectors, called ultrasound transducers [16], which can convert mechanical stresses into electrical signals, are placed around the tissue to record these outgoing acoustic waves, commonly 
referred to as thermoacoustic or photoacoustic signals. These thermoacoustic signals carry information about the RF absorption or initiated stress as well as about the acoustic properties of the tissue. Since the RF absorption or initiated stress is directly related to certain tissue properties (i.e., ionic conductivity and water components, etc.), the key problem is how to reconstruct the distribution of the RF absorption or initiated stress from the measured thermoacoustic signals around the tissue surface.

The short duration of the RF pulse allows one to restrict the RF energy deposition within the absorbing volume and minimize the thermal diffusion effect on the thermoacoustic waves. In thermoacoustic imaging, the RF pulse duration, $\tau_{p}$, is typically shorter than the thermal transport time of absorbed RF energy in thermal conduction, $\tau_{\text {th }}$, the condition that is commonly referred to as thermal confinement [17]. The condition for thermal confinement can be expressed as $\tau_{p}<\tau_{\text {th }} \sim l_{p}^{2} / \alpha$, where $\alpha$ is the thermal diffusivity of the irradiated material and $l_{p}$ is the RF penetration depth or the size of the absorbing structure. For most soft tissues, $\alpha \sim 10^{3} \mathrm{~cm}^{2} \cdot \mathrm{s}^{-1}$ [14]. For example, we are interested in the detection of small absorbers in sizes from submillimeters to centimeters inside the tissue. We choose $l_{p} \sim$ mm to underestimate the thermal transport time $\tau_{\text {th }} \sim 10 \mu \mathrm{s}$. The RF pulse used, $\tau_{p}$, is typically less than $1 \mu \mathrm{s}$, which is much less than $\tau_{\mathrm{th}}$. Moreover, the time required for an acoustic wave to traverse the absorption depth $l_{p}$ approximates to $\sim l_{p} / c \approx 0.7 \mu \mathrm{s}$, which is also much shorter than $\tau_{\text {th }}$, where $c$ is the sound speed that is around $1.5 \mathrm{~mm} / \mu \mathrm{s}$ in most soft tissues [14]. In other words, even in $1 \mu$ s of RF pulse duration, the heat transports a length of $\sqrt{\alpha \tau_{p}} \sim 0.3 \mathrm{~mm}$, while in the same amount of time, the acoustic wave propagates a distance of $c \tau_{p} \sim 1.5 \mathrm{~mm}$, which is far away from the thermal diffusion region of $0.3 \mathrm{~mm}$. Of course, thermal diffusion will slightly blur the reconstructed images. But, when we try to investigate targets that are bigger than the thermal diffusion region, for instance, $>0.3 \mathrm{~mm}$, if the RF pulse duration is less than $1 \mu \mathrm{s}$, the thermal effect on the thermoacoustic waves in soft tissue can be ignored. In addition, the thermoacoustic signal excited by a RF pulse with finite width can be regarded as a convolution with the RF pulse profile and the thermoacoustic signal excited by a $\delta(t) \mathrm{RF}$ irradiation. For theoretical analysis, the short pulse can be regarded as a delta function.

In general, thermoacoustic imaging can be used for the investigation of soft tissues with inhomogeneous RF absorption but relatively homogeneous acoustic properties including the speed of sound and low acoustic attenuation. For practical purposes, speed dispersion can be neglected in soft tissues; typically, the speed increases by about $0.01 \% \mathrm{MHz}^{-1}$ [16]. In most soft tissues, the speed of sound is relatively constant at $\sim 1.5 \mathrm{~mm} / \mu \mathrm{s}$ with a small variation about 5\% [14], [16]. Acoustic attenuation in soft tissues is primarily due to the spectra of the relaxation processes, which account for the nearly linear frequency dependence [16]. The total acoustic attenuation in soft tissues results from combined losses due to absorption and scattering [14], [16]. In the low megahertz range, acoustic scattering in soft tissues accounts for only about $10 \%$ of the total acoustic attenuation [14]. A mean value of the acoustic energy attenuation in soft tissue is equal to $0.6 \mathrm{~dB} \cdot \mathrm{cm}^{-1} \cdot \mathrm{MHz}^{-1}$ [16]. Typically, the total energy attenuation for a $1-\mathrm{MHz}$ signal after a $5-\mathrm{cm}$ prop- agation is about $3 \mathrm{~dB}$, and the corresponding amplitude attenuates approximately to $70 \%$ of the initial value. Such attenuation is still acceptable, although the spatial resolution will be blurred at a certain level due to the loss of the high-frequency signal. For simplicity, the acoustic attenuation is neglected here. Pure acoustic property differentiation should appeal to conventional ultrasound imaging [16]. The unique advantage of thermoacoustic imaging is its ability to detect the inhomogeneous RF absorption property of tissues when the acoustic property is homogeneous. An obvious application is the detection of breast cancer tumors. People have observed that tumors in the breast have a stronger rate of RF absorption than the surrounding tissues; by contrast, the ultrasonic contrast in soft tissues is quite low [8].

In previous papers [2]-[4], the authors have presented studies on scanning thermoacoustic tomography using focused ultrasonic transducers as in conventional pulse-echo ultrasound imaging [16]. Each scan line is converted into a one-dimensional (1-D) image along the axis of the focused transducer, and only a simple calculation is required to construct cross-sectional images from all of the scan lines. However, the lateral resolution of this approach is determined by the focal diameter of the transducer as with conventional ultrasound, and the imaging region is also limited to the focal length of the transducer. To obtain a larger imaging view, we use unfocused wide-band point transducers to record the thermoacoustic signals. In this approach, a complicated reconstruction algorithm has to be derived for computing the images from a set of data measured around the tissue under study. Different recording geometric configurations result in different reconstruction formulas.

The puzzle of finding good reconstruction algorithms has not yet been resolved. Some researchers have resorted to approximated reconstruction algorithms, such as the Radon transform in the far-field approximation [7], [9], the weighted delay-and-sum method with experiential weighting factors [10], or the optimal statistical approach [18]. To date, some rigorous reconstruction algorithms have been reported for idealized measurement configurations, such as for the fully enclosing spherical recording surface [5], the planar recording surface of an infinite extent [19], [20] and the cylindrical recording surface of an infinite length [21]. However, in practical applications, the recording surfaces are generally finite and partially enclosing.

In this paper, we will first discuss the inverse problem of thermoacoustic imaging. Then, by employing the Green's function technique, we will generalize the rigorous reconstruction formulas for three types of recording surfaces: a planar, a spherical, and a cylindrical surface, which enclose the sample under study. In order to detect small but relatively deeply buried targets, we will introduce the following two conditions (details given in Section II): the high-frequency components of thermoacoustic signals contribute more to spatial resolution than the low-frequency ones, and the detecting distances between the thermoacoustic sources and the detecting transducers are much larger than the wavelengths of the high-frequency thermoacoustic signals that are useful for imaging. Taking these conditions into account, we will simplify the rigorous formulas and present time-domain reconstruction algorithms, which can be computed by temporal back projections and coherent summations over 
spherical surfaces with certain spatial weighting factors. Finally, numerical experiments will be conducted to demonstrate the validity of these formulas.

\section{INVERSE PROBLEM}

As discussed in Section I, in typical thermoacoustic measurements, the RF pulse duration is so short that the thermal conduction time is far greater than the thermoacoustic transit time and the effect of thermal diffusion on the thermoacoustic wave in the tissue can be ignored. We focus on small-amplitude thermoacoustic propagation using safe levels of RF irradiation. Thus, the inverse problem that we want to solve is a linear acoustic-wave equation.

The pressure $p(\mathbf{r}, t)$ at position $\mathbf{r}$ and time $t$ in an acoustically homogeneous medium in response to a heat source $H(\mathbf{r}, t)$ obeys the following equation [5], [23]:

$$
\nabla^{2} p(\mathbf{r}, t)-\frac{1}{c^{2}} \frac{\partial^{2}}{\partial t^{2}} p(\mathbf{r}, t)=-\frac{\beta}{C_{p}} \frac{\partial}{\partial t} H(\mathbf{r}, t)
$$

where $C_{p}$ is the specific heat, $H(\mathbf{r}, t)$ is the heating function defined as the thermal energy deposited by the energy source per time and volume, $\beta$ is the isobaric volume expansion coefficient, and $c$ is the speed of sound. The heating function can be written as the product of a spatial absorption function and a temporal illumination function of the RF source

$$
H(\mathbf{r}, t)=A(\mathbf{r}) I(t) .
$$

As discussed in Section I, the short RF pulse can be regarded as a Dirac delta function

$$
I(t)=\delta(t)
$$

Substituting (2) and (3) into (1) and taking the Fourier transform on variable $\bar{t}=c t$ of (1), one gets

$$
\left(\nabla^{2}+k^{2}\right) \widetilde{p}(\mathbf{r}, k)=i k c^{2} \eta A(\mathbf{r})
$$

where $\eta=\beta / C_{p}$, and the following Fourier transform pair exists:

$$
\begin{aligned}
& \widetilde{p}(\mathbf{r}, k)=\int_{-\infty}^{+\infty} p(\mathbf{r}, \bar{t}) \exp (i k \bar{t}) d \bar{t}, \\
& p(\mathbf{r}, \bar{t})=\frac{1}{2 \pi} \int_{-\infty}^{+\infty} \widetilde{p}(\mathbf{r}, k) \exp (-i k \bar{t}) d k
\end{aligned}
$$

where the acoustic wave number $k=\omega / c$ and $\omega$ is the angular frequency and equal to $2 \pi f$; and $\widetilde{p}(\mathbf{r}, k)$ is the frequency spectrum of the thermoacoustic signal $p(\mathbf{r}, \bar{t})$. Equation (4) is a nonhomogeneous Helmholtz equation. Assume that the thermoacoustic signals are measured on a surface $S_{0}$ that encloses the sample under study, the frequency spectrum of the thermoacoustic pressure measured at the position $\mathbf{r}_{0}$ on surface $S_{0}$ can be expressed as [22]

$$
\widetilde{p}\left(\mathbf{r}_{0}, k\right)=-i k c^{2} \eta \iint_{V} \int d^{3} r A(\mathbf{r}) \widetilde{G}_{k}\left(\mathbf{r}, \mathbf{r}_{0}\right)
$$

where $\widetilde{G}_{k}\left(\mathbf{r}, \mathbf{r}_{0}\right)$ is the Green's function of the nonhomogeneous equation

$$
\left(\nabla^{2}+k^{2}\right) \widetilde{G}_{k}\left(\mathbf{r}, \mathbf{r}_{0}\right)=-\delta\left(\mathbf{r}-\mathbf{r}_{0}\right) .
$$

In general, Green's function in three dimensions can be written as [22]

$$
\widetilde{G}_{k}\left(\mathbf{r}, \mathbf{r}_{0}\right)=\frac{\exp \left(i k\left|\mathbf{r}-\mathbf{r}_{0}\right|\right)}{4 \pi\left|\mathbf{r}-\mathbf{r}_{0}\right|}
$$

Now, the inverse problem is to reconstruct the absorption distribution $A(\mathbf{r})$ from a set of data $p\left(\mathbf{r}_{0}, t\right)$ or $\widetilde{p}\left(\mathbf{r}_{0}, k\right)$ measured at position $\mathbf{r}_{0}$. Equation (7) shows a linear mapping connecting $A(\mathbf{r})$ and $\widetilde{p}\left(\mathbf{r}_{0}, k\right)$. The solution of $A(\mathbf{r})$ can be expected in a similar form- - linear integral

$$
A(\mathbf{r})=\iint_{S_{0}} d S_{0} \int_{k} d k \widetilde{p}\left(\mathbf{r}_{0}, k\right) \widetilde{K}_{k}\left(\mathbf{r}_{0}, \mathbf{r}\right)
$$

where $d S_{0}=d^{2} \mathbf{r}_{0}, S_{0}$ is the total recording surface, and the integral kernel $\widetilde{K}_{k}\left(\mathbf{r}_{0}, \mathbf{r}\right)$ needs to be determined. As shown in Section III, the integral kernel is complicated. But under most practical conditions, as discussed below, it can be simplified to a linear relation with the Green's function.

The greatest challenge is to detect small (compared with measurement geometry) but deeply buried targets inside the tissue. Let us check the property of the frequency spectrum of acoustic waves generated from a small object. Assume there is a homogeneous $\mathrm{RF}$ absorption sphere with a size of $2 a$ in diameter, i.e., the spatial absorption function $A(\mathbf{r})=U(a-r)$, where the step function $U(\xi)=1, \xi \geq 0$ and $U(\xi)=0, \xi<0$. With a $\delta(t) \mathrm{RF}$ illumination, the radiated acoustic wave from this sphere can be expressed as $p(r, t)=\eta c^{2} U(a-|r-c t|)(r-c t) /(2 r)$ [23]. Applying the Fourier transform gives the frequency spectrum $\sim j_{1}(k a)$, where $j_{1}(k a)$ is the spherical Bessel function of the first kind. The main beam of the above spectrum is in a belly shape with maximum amplitude at the central frequency $f_{c} \approx 0.7 c /(2 a)$. For example, for an object with a size of 1 $\mathrm{mm}, f_{c}=0.7 \times 1.5(\mathrm{~mm} / \mu \mathrm{s}) /(1 \mathrm{~mm}) \approx 1 \mathrm{MHz}$. Below $100 \mathrm{KHz}$, the spectrum amplitude is less than 0.1 of the maximum value, and particularly at $0 \mathrm{~Hz}$, the spectrum amplitude is zero, which can be proved using (7) letting $k=0$. In general, the frequency spectrum of acoustic waves generated from a small object concentrates in the relatively high-frequency region. The dominating frequency or central frequency $f_{c}$ can be approximated by the reciprocal of the required time $\tau$ for an acoustic wave to traverse the object length $l$, i.e., $f_{c} \approx 1 / \tau=$ $c / l$. In addition, the boundaries of large objects can also be regarded as small structures, which are also determined by relatively high-frequency signals. In other words, only the relatively high-frequency thermoacoustic signals can restore small absorbers as well as the boundaries of big absorbers.

During measurement, the transducer for ultrasonic imaging [16] can be employed to receive thermoacoustic signals. The ideal transducer for receiving ultrasound would have a wide dynamic range and a wide frequency response. Most commonly, transducers are operated over a band of frequencies containing 
a resonant frequency, which is determined by the physical property of the transducer [16]. A transducer with a resonant or central frequency of 1-3 MHz could be perfectly matched to millimeter-sized small absorbers in soft tissues. The real-time localization of targets should employ transducer arrays, in which all of the small elements serve independent ultrasound detectors and simultaneously receive thermoacoustic signals at different positions around the investigated tissue [16]. Currently, a linear or circular array with hundreds of small elements, in which each element has a size of $\sim$ sub-mm with a total length of perhaps $\sim 10 \mathrm{~cm}$, is available on the market or can be customized and manufactured in a research lab [16]. In addition, the measurement geometry is relatively big compared with the small targets. For example, when using a spherical measurement configuration with a radius $r_{0}=5 \mathrm{~cm}$, even at $f=100 \mathrm{KHz}$, $k r_{0} \approx 20 \gg 1$. In another example, for a target inside a tissue with a distance to the nearest detection element $d=1 \mathrm{~cm}$, at $f=1 \mathrm{MHz}, k d \approx 40 \gg 1$ and even at $f=100 \mathrm{KHz}$, $k d \approx 4>1$.

Therefore, for practical applications, we introduce the following two conditions: the high-frequency components of the thermoacoustic signals contribute more to the spatial resolution than the low-frequency ones, and the detecting distances between the thermoacoustic sources and the detecting transducers are much larger than the wavelengths of the high-frequency thermoacoustic signals. Taking these conditions into account, we will simplify the rigorous formulas and present time-domain reconstruction algorithms in the following sections.

\section{RECONSTRUCTION FORMULAS}

\section{A. Planar Measurement Configuration}

The Cartesian coordinate system $\mathbf{r}=(x, y, z)$ suits this situation. As shown in Fig. 1(a), we assume that the measurement surface is the $z=0$ plane, i.e., $\mathbf{r}_{0}=\left(x_{0}, y_{0}, 0\right)$. The sample lies above the plane, i.e., $A(\mathbf{r})=A(x, y, z)$ where $z>0$ and $A(\mathbf{r})=0$ when $z<0$. Taking Fourier transforms on both sides of (8) on variables $x, y$ and $z$, it can be shown that the Green's function is a triple Fourier integral [22]

$$
\begin{aligned}
\widetilde{G}_{k}\left(\mathbf{r}, \mathbf{r}_{0}\right) & =\frac{1}{(2 \pi)^{3}} \iint_{-\infty}^{+\infty} \int d K_{x} d K_{y} d K_{z} \\
. & \frac{\exp \left[i K_{x}\left(x_{0}-x\right)+i K_{y}\left(y_{0}-y\right)-i K_{z} z\right]}{K_{x}^{2}+K_{y}^{2}+K_{z}^{2}-k^{2}} .
\end{aligned}
$$

Considering the above expansion, and referencing the mathematical techniques in Norton's work on ultrasonic reflectivity imaging [24], we can derive a rigorous reconstruction formula in the form of (10) as (see Appendix A)

$$
A(x, y, z)=\int_{-\infty}^{+\infty} \int_{-\infty}^{+\infty} d x_{0} d y_{0} \int_{-\infty}^{+\infty} d k \widetilde{p}\left(x_{0}, y_{0}, k\right) \widetilde{K}_{k}\left(\mathbf{r}_{0}, \mathbf{r}\right)
$$

with

$$
\begin{aligned}
\widetilde{K}_{k}\left(\mathbf{r}_{0}, \mathbf{r}\right)= & \frac{1}{4 \pi^{3} c^{2} \eta} \int_{\rho=0}^{\rho=|k|} d u d v \exp \left[-i z \operatorname{sgn}(k) \sqrt{k^{2}-\rho^{2}}\right] \\
& \cdot \exp \left[i u\left(x_{0}-x\right)+i v\left(y_{0}-y\right)\right]
\end{aligned}
$$

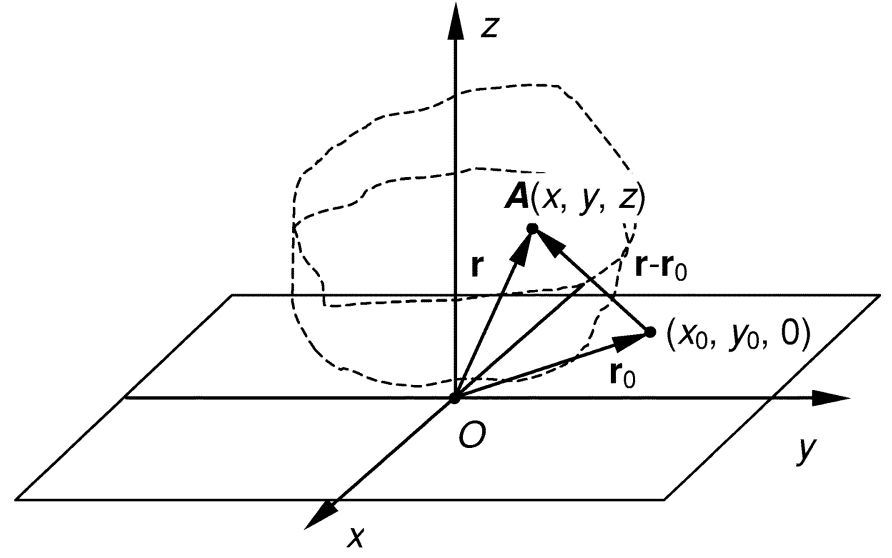

(a)

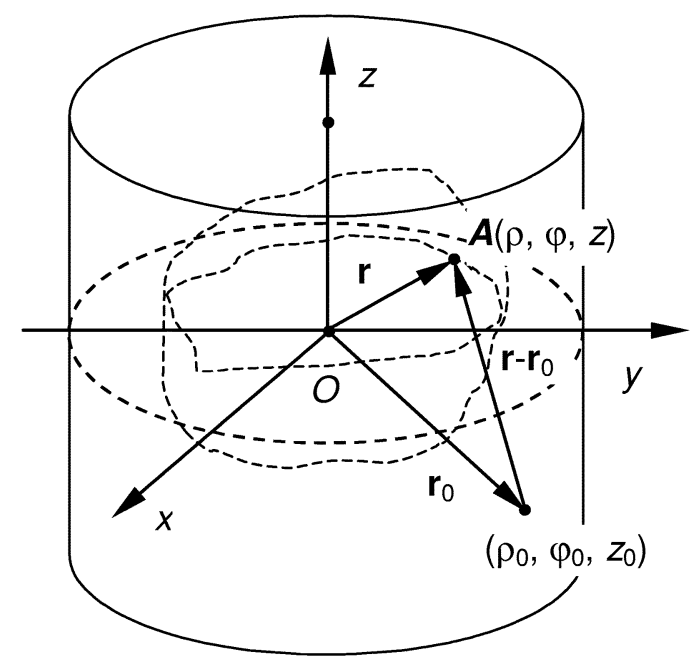

(b)

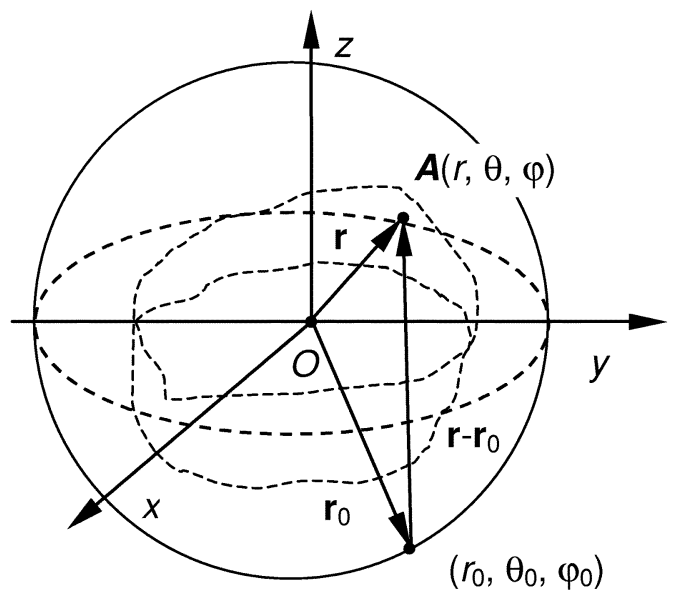

(c)

Fig. 1. Diagram of the measurement: (a) planar measurement configuration, (b) cylindrical measurement configuration, and (c) spherical measurement configuration.

where $\rho=\sqrt{u^{2}+v^{2}}$, and the sign function: $\operatorname{sgn}(k)=1$ if $k>0$, and $\operatorname{sgn}(k)=-1$ if $k<0$.

Under the condition $|k|\left|\mathbf{r}-\mathbf{r}_{0}\right| \gg 1$, which means that the detecting distances between the thermoacoustic sources and the detecting transducers are much greater than the wavelengths of 
the thermoacoustic signals that are useful for imaging, (13) reduces to (see Appendix A)

$$
\widetilde{K}_{k}\left(\mathbf{r}_{0}, \mathbf{r}\right) \approx \frac{i 2 k}{\pi c^{2} \eta} \frac{z}{\left|\mathbf{r}-\mathbf{r}_{0}\right|} \widetilde{G}_{k}^{*}\left(\mathbf{r}, \mathbf{r}_{0}\right)
$$

where " $*$ " stands for the complex conjugate.

It can be shown that $\mathbf{n} \cdot \mathbf{n}_{0}=z /\left|\mathbf{r}-\mathbf{r}_{0}\right|$, where $\mathbf{n}$ and $\mathbf{n}_{0}$ are unit vectors pointing along the $z$ axis and along the line joining $\mathbf{r}$ and $\mathbf{r}_{0}$, respectively. Substituting (14) into (12), we get

$$
\begin{aligned}
A(\mathbf{r})= & \iint_{S_{0}} d S_{0} \int_{-\infty}^{+\infty} d k \frac{i 2 k}{\pi c^{2} \eta} \widetilde{p}\left(\mathbf{r}_{0}, k\right) \widetilde{G}_{k}^{*}\left(\mathbf{r}, \mathbf{r}_{0}\right)\left[\mathbf{n} \cdot \mathbf{n}_{0}\right] \\
= & -\frac{1}{\pi c^{2} \eta} \iint_{S_{0}} d S_{0} \cdot \frac{1}{2 \pi} \int_{-\infty}^{+\infty} d k(-i k) \widetilde{p}\left(\mathbf{r}_{0}, k\right) \\
& \times \frac{\exp \left(-i k\left|\mathbf{r}-\mathbf{r}_{0}\right|\right)}{\left|\mathbf{r}-\mathbf{r}_{0}\right|}\left[\mathbf{n} \cdot \mathbf{n}_{0}\right]
\end{aligned}
$$

where $d S_{0}=d x_{0} d y_{0}$. Recalling the inverse Fourier transform of (6), (15) reduces to

$$
A(\mathbf{r})=-\left.\frac{1}{\pi c^{4} \eta} \iint_{S_{0}} d S_{0}\left[\mathbf{n} \cdot \mathbf{n}_{0}\right] \frac{1}{t} \frac{\partial p\left(\mathbf{r}_{0}, t\right)}{\partial t}\right|_{t=\frac{\left|\mathbf{r}_{0}-\mathbf{r}\right|}{c}} .
$$

This is a modified back projection formula of quantity $-(1 / t)\left(\partial p\left(\mathbf{r}_{0}, t\right) / \partial t\right)$ with a weighting factor $\left[\mathbf{n} \cdot \mathbf{n}_{0}\right]$. The required condition is $|k|\left|\mathbf{r}-\mathbf{r}_{0}\right|>>1$.

\section{B. Cylindrical Measurement Configuration}

In this case, a circular cylindrical coordinate system $\mathbf{r}=$ $(\rho, \varphi, z)$ is convenient. As shown in Fig. 1(b), we assume that the measurement surface is a circular cylindrical surface $\mathbf{r}_{0}=$ $\left(\rho_{0}, \varphi_{0}, z_{0}\right)$. The sample (of a finite size) lies inside the cylinder. The Green's function can be expressed in the cylindrical coordinates $(k>0)$ (see Appendix B for detail)

$$
\begin{aligned}
\widetilde{G}_{k}\left(\mathbf{r}, \mathbf{r}_{0}\right)= & \frac{1}{4 \pi^{2}} \sum_{m=-\infty}^{+\infty} \exp \left[i m\left(\varphi-\varphi_{0}\right)\right] \\
& \cdot \int_{-\infty}^{+\infty} d k_{z} \exp \left[i k_{z}\left(z-z_{0}\right)\right] g_{m k}\left(\rho, \rho_{0}, k_{z}\right)
\end{aligned}
$$

where if $k_{z}^{2}<k^{2}, g_{m k}\left(\rho, \rho_{0}, k_{z}\right)=(i \pi / 2) J_{m}(\mu \rho) H_{m}^{(1)}\left(\mu \rho_{0}\right)$ with $\mu=\sqrt{k^{2}-k_{z}^{2}}$; if $k_{z}^{2}>k^{2}, g_{m k}\left(\rho, \rho_{0}, k_{z}\right)=$ $I_{m}(-i \mu \rho) K_{m}\left(-i \mu \rho_{0}\right)$ with $\mu=i \sqrt{k_{z}^{2}-k^{2}} . J_{m}(\cdot), H_{m}^{(1)}(\cdot)$, $I_{m}(\cdot)$ and $K_{m}(\cdot)$ are the Bessel function of the first kind, the Hankel function of the first kind, the modified Bessel function of the first kind, and the modified Bessel function of the second kind, respectively.

After some deduction (see Appendix B), we get the reconstruction formula in the form of (10) as

$$
A(\rho, \varphi, z)=\iint_{S_{0}} d S_{0} \int_{0}^{+\infty} d k \widetilde{p}\left(\mathbf{r}_{0}, k\right) \widetilde{K}_{k}\left(\mathbf{r}_{0}, \mathbf{r}\right)
$$

where $d S_{0}=\rho_{0} d \varphi_{0} d z_{0}$, and

$$
\begin{aligned}
\widetilde{K}_{k}\left(\mathbf{r}_{0}, \mathbf{r}\right) & =\frac{1}{2 \pi^{3} c^{2} \eta \rho_{0}} \int_{-k}^{+k} d \gamma \exp \left[i \gamma\left(z_{0}-z\right)\right] \\
& \cdot \sum_{n=-\infty}^{+\infty} \exp \left[i n\left(\varphi_{0}-\varphi\right)\right] \frac{J_{n}\left(\rho \sqrt{k^{2}-\gamma^{2}}\right)}{H_{n}^{(1)}\left(\rho_{0} \sqrt{k^{2}-\gamma^{2}}\right)} .
\end{aligned}
$$

Under the conditions introduced in Section II, i.e., $\rho_{0} k \gg 1$, (19) approximates to (see Appendix B)

$$
\widetilde{K}_{k}\left(\mathbf{r}_{0}, \mathbf{r}\right) \approx \frac{i 2 k}{\pi c^{2} \eta} \sqrt{1-\frac{\left(z_{0}-z\right)^{2}}{\left|\mathbf{r}-\mathbf{r}_{0}\right|^{2}}} \widetilde{G}_{k}^{*}\left(\mathbf{r}, \mathbf{r}_{0}\right) .
$$

Adding the complex conjugate of (18) onto itself and then dividing the summation by two, and further considering $\widetilde{p}^{*}\left(\mathbf{r}_{0}, k\right)=\widetilde{p}\left(\mathbf{r}_{0},-k\right)$ and the approximation (20), one gets

$$
\begin{aligned}
A(\rho, \varphi, z)= & \iint_{S_{0}} d S_{0} \int_{-\infty}^{+\infty} d k \frac{i k}{c^{2} \eta \pi} \widetilde{p}\left(\mathbf{r}_{0}, k\right) \\
& \cdot \frac{\exp \left(-i k\left|\mathbf{r}-\mathbf{r}_{0}\right|\right)}{4 \pi\left|\mathbf{r}-\mathbf{r}_{0}\right|} \sqrt{1-\frac{\left(z_{0}-z\right)^{2}}{\left|\mathbf{r}-\mathbf{r}_{0}\right|^{2}}} \\
= & -\frac{1}{2 \pi c^{2} \eta} \iint_{S_{0}} d S_{0} \sqrt{1-\frac{\left(z_{0}-z\right)^{2}}{\left|\mathbf{r}-\mathbf{r}_{0}\right|^{2}}} \\
& \cdot \frac{1}{2 \pi} \int_{-\infty}^{+\infty}(-i k) d k \widetilde{p}\left(\mathbf{r}_{0}, k\right) \frac{\exp \left(-i k\left|\mathbf{r}-\mathbf{r}_{0}\right|\right)}{\left|\mathbf{r}-\mathbf{r}_{0}\right|}
\end{aligned}
$$

It can be shown that

$$
\begin{aligned}
\mathbf{n} \cdot \mathbf{n}_{0} & =\frac{\left|\boldsymbol{\rho}-\boldsymbol{\rho}_{0}\right|}{\left|\mathbf{r}-\mathbf{r}_{0}\right|} \\
& =\sqrt{\frac{\rho^{2}+\rho_{0}^{2}-2 \rho \rho_{0} \cos \left(\varphi_{0}-\varphi\right)}{\left|\mathbf{r}-\mathbf{r}_{0}\right|^{2}}} \\
& =\sqrt{1-\frac{\left(z_{0}-z\right)^{2}}{\left|\mathbf{r}-\mathbf{r}_{0}\right|^{2}}}
\end{aligned}
$$

where $\rho$ and $\rho_{0}$ are the projections of $\mathbf{r}$ and $\mathbf{r}_{0}$ on $z$ plane, respectively, and $\mathbf{n}$ and $\mathbf{n}_{0}$ are unit vectors pointing along the line joining $\boldsymbol{\rho}$ and $\boldsymbol{\rho}_{0}$ and along the line joining $\mathbf{r}$ and $\mathbf{r}_{0}$, respectively. Recalling the inverse transformation (6), we can rewrite (21) as

$$
A(\rho, \varphi, z)=-\left.\frac{1}{2 \pi c^{4} \eta} \iint_{S_{0}} d S_{0}\left[\mathbf{n} \cdot \mathbf{n}_{0}\right] \frac{1}{t} \frac{\partial p\left(\mathbf{r}_{0}, t\right)}{\partial t}\right|_{t=\frac{\left|\mathbf{r}-\mathbf{r}_{0}\right|}{c}} .
$$

This is a modified back projection formula of quantity $-(1 / t)\left(\partial p\left(\mathbf{r}_{0}, t\right) / \partial t\right)$ with a weighting factor $\left[\mathbf{n} \cdot \mathbf{n}_{0}\right]$. The required condition is $\rho_{0}|k| \gg 1$ and $|k|\left|\mathbf{r}-\mathbf{r}_{0}\right| \gg 1$.

\section{Spherical Measurement Configuration}

This instance has been reported in another paper [5]. As a consequence, we only briefly review the results here for completeness. 
We use the spherical polar coordinate system $\mathbf{r}=(r, \theta, \varphi)$. As shown in Fig. 1(c), we assume that the recording surface is a spherical surface $\mathbf{r}_{0}=\left(r_{0}, \theta_{0}, \varphi_{0}\right)$. The sample lies inside the sphere, i.e., $A(\mathbf{r})=A(r, \theta, \varphi)$ where $r<r_{0}$ and $A(\mathbf{r})=0$ when $r>r_{0}$. The Green's function can be expanded as a series based on the spherical Bessel function of the first kind $j_{l}(\cdot)$, the spherical Hankel function of the first kind $h_{l}(\cdot)$, and the Legendre polynomial $P_{l}(\cdot)$

$\widetilde{G}_{k}\left(\mathbf{r}, \mathbf{r}_{0}\right)=\frac{i k}{4 \pi} \sum_{l=0}^{\infty}(2 l+1) j_{l}(k r) h_{l}^{(1)}\left(k r_{0}\right) P_{l}\left(\mathbf{n} \cdot \mathbf{n}_{0}\right),(k>0)$

where $\mathbf{n}=\mathbf{r} / r$, and $\mathbf{n}_{0}=\mathbf{r}_{0} / r_{0}$.

We find the rigorous reconstruction formula as

$$
A(r, \theta, \varphi)=\iint_{S_{0}} d S_{0} \int_{0}^{+\infty} d k \widetilde{p}\left(\mathbf{r}_{0}, k\right) \widetilde{K}_{k}\left(\mathbf{r}_{0}, \mathbf{r}\right)
$$

where $d S_{0}=r_{0}^{2} \sin \theta_{0} d \theta_{0} d \varphi_{0}$, and

$$
\widetilde{K}_{k}\left(\mathbf{r}_{0}, \mathbf{r}\right)=\frac{1}{2 \pi^{2} c^{2} \eta r_{0}^{2}} \sum_{m=0}^{\infty} \frac{(2 m+1) j_{m}(k r)}{h_{m}^{(1)}\left(k r_{0}\right)} P_{m}\left(\mathbf{n} \cdot \mathbf{n}_{0}\right) .
$$

Under the condition $k r_{0} \gg 1$, one can approximate

$$
\widetilde{K}_{k}\left(\mathbf{r}_{0}, \mathbf{r}\right) \approx \frac{i 2 k}{\pi c^{2} \eta} \widetilde{G}_{k}^{*}\left(\mathbf{r}, \mathbf{r}_{0}\right)
$$

Adding the complex conjugate of (25) onto itself and then dividing the summation by two, and further considering $\widetilde{p}^{*}\left(\mathbf{r}_{0}, k\right)=\widetilde{p}\left(\mathbf{r}_{0},-k\right)$ and the approximation (27), we get

$$
\begin{aligned}
A(r, \theta, \varphi)= & -\frac{1}{2 \pi c^{2} \eta} \iint_{S_{0}} d S_{0} \\
& \cdot \frac{1}{2 \pi} \int_{-\infty}^{+\infty} d k \widetilde{p}\left(\mathbf{r}_{0}, k\right)(-i k) \frac{\exp \left(-i k\left|\mathbf{r}_{0}-\mathbf{r}\right|\right)}{\left|\mathbf{r}_{0}-\mathbf{r}\right|} .
\end{aligned}
$$

Recalling the inverse Fourier transform (6), (28) reduces to

$$
A(r, \theta, \varphi)=-\left.\frac{1}{2 \pi c^{4} \eta} \iint_{S_{0}} d S_{0} \frac{1}{t} \frac{\partial p\left(\mathbf{r}_{0}, t\right)}{\partial t}\right|_{t=\frac{\left|\mathbf{r}_{0}-\mathbf{r}\right|}{c}} .
$$

Equation (29) shows that the absorption distribution can be calculated by means of back projection of the quantity $-(1 / t)\left(\partial p\left(\mathbf{r}_{0}, t\right) / \partial t\right)$. The required condition is $|k| r_{0} \gg 1$.

As expected, all of the reconstruction formulas-(16) for the planar measurement configuration, (23) for the cylindrical measurement configuration, and (29) for the spherical measurement configuration-can be carried out in the time domain. They share a similar expression, except for the weighting factor [n . $\mathbf{n}_{0}$ ]. These formulas can be referred to as modified back-projections. Compared with (16), (23) and (29) have an additional factor $1 / 2$. This is because the planar measurement configuration can cover a solid angle of up to $2 \pi$ only while the other two configurations can cover a full $4 \pi$ solid angle.

\section{NUMERICAL EXPERIMENTS}

Now we want to conduct some numerical experiments to demonstrate the validity of the above time-domain reconstruction formulas for thermoacoustic imaging.

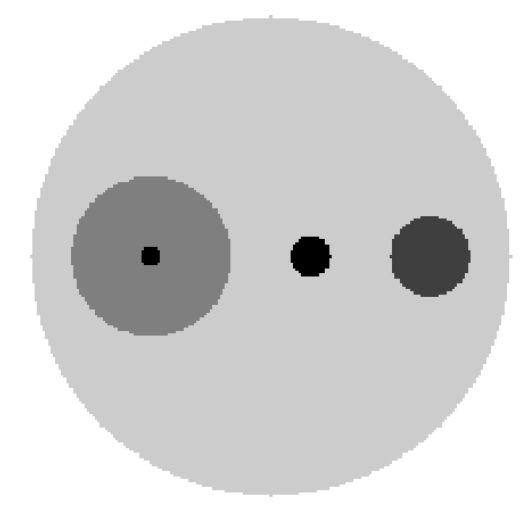

(a)

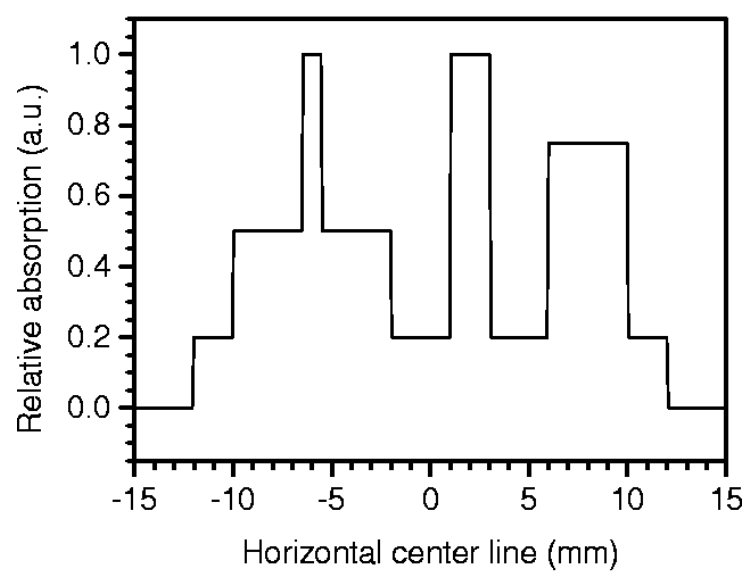

(b)

Fig. 2. Original sample. (a) Cross-sectional image. (b) Profile along the horizontal center line.

We consider uniform spherical absorbers surrounded by a nonabsorbing background medium. For convenience, we use the centers of the absorbers to denote their positions. The uniform spherical absorber can be written as $A(\mathbf{r})=A_{0} U\left(a-\left|\mathbf{r}-\mathbf{r}_{a}\right|\right)$, where $A_{0}$ is the absorption intensity, and $a$ and $\mathbf{r}_{a}$ are the radius and the center of the sphere, respectively. As shown in Fig. 2(a), assume a sample contains five spherical absorbers with different absorption intensities and the centers of these spheres lie in a line parallel to the $x$ axis. For convenience, we call this line the horizontal center line. As shown in Fig. 2(b), from the smallest to the biggest, the radii are $0.5,1,2,4$, and $12 \mathrm{~mm}$, respectively, and the relative absorption intensities are 1, 1, 0.75, 0.5, and 0.2 , respectively. We also assume that the RF pulse duration is very short and can be regarded as a delta function, and, consequently, that the thermoacoustic signal $p\left(\mathbf{r}_{0}, t\right)$ irradiated from a uniform sphere can be calculated by $\eta c^{2} U(a-|R-c t|)(R-$ $c t) /(2 R)$, where $R$ is the distance between the detection position $\mathbf{r}_{0}$ and the absorber center $\mathbf{r}_{a}\left(R=\left|\mathbf{r}_{0}-\mathbf{r}_{a}\right|\right)$ [23]. The quantity $\partial p\left(\mathbf{r}_{0}, t\right) / \partial t$ in the reconstruction formulas (16), (23), and (29) can be calculated through the Fourier transform

$$
\frac{\partial p\left(\mathbf{r}_{0}, t\right)}{\partial t}=\operatorname{IFFT}\left\{-i \omega p\left(\mathbf{r}_{0}, \omega\right) W_{\Omega}(\omega)\right\}
$$


where IFFT denotes the inverse fast Fourier transform, $W_{\Omega}(\omega)$ is a window function, and the Fourier transform defines

$$
\bullet(\omega)=\int_{-\infty}^{+\infty} \bullet(t) \exp (i \omega t) d t
$$

As we discussed in [6], the factor $\omega$ in (30) actually represents a pure ramp filter, which will significantly depress the low-frequency signal. It is helpful for guaranteeing the validity of the reconstruction (16), (23), and (29). It also indicates that the relatively high-frequency component of the signals play the primary role in the restoration of the RF absorption distribution inside the tissue. But, the ramp filter can also amplify the high-frequency noise in such a way that the reconstructed image is not acceptable from the physical point of view. In order to avoid this effect, it is necessary to introduce a relatively low-pass filter $W_{\Omega}(\omega)$ characterized by a cutoff angular frequency $\Omega=2 \pi f_{\Omega}$. A Hanning window is our choice in this case

$$
W_{\Omega}(\omega)= \begin{cases}0.5+0.5 \cos \left(\pi \frac{\omega}{\Omega}\right), & \text { if }|\omega|<\Omega, \\ 0, & \text { otherwise. }\end{cases}
$$

In addition, $W_{\Omega}(\omega)$ also reflects the limited bandwidth of the detected thermoacoustic signals that is due to the finite bandwidth of the detector. We assume the thermoacoustic waves to be in a frequency range below $4 \mathrm{MHz}$, and choose $f_{\Omega}=4 \mathrm{MHz}$; then the dominative frequency in $\omega W_{\Omega}(\omega)$ is $1.7 \mathrm{MHz}$. Here, the data sampling frequency is $20 \mathrm{MHz}$.

\section{A. Planar Measurement Configuration}

We use the planar measurement configuration as shown in Fig. 1(a). Assume that the measurement area is $120 \mathrm{~mm} \times 120$ $\mathrm{mm}$ in the $z=0$ plane and that the thermoacoustic signals are collected at 3600 total detection positions that are evenly distributed in the measurement area. Such a measurement can be realized by using a rectangular ultrasonic array or by scanning a linear array or even by scanning a single detector to cover the measurement area. The center of the measurement area is $(0$, $0,0)$. The sample center $(0,0,30)$ lies $30 \mathrm{~mm}$ above the measurement area. Fig. 3(a) shows the reconstructed RF absorption distribution of the $z=30 \mathrm{~mm}$ plane, and Fig. 3(b) shows the comparison of the original and reconstructed absorption profiles along the horizontal center line.

\section{B. Cylindrical Measurement Configuration}

We employ the cylindrical measurement configuration as shown in Fig. 1(b). Assume the measurement area is a cylindrical surface with a length of $90 \mathrm{~mm}$ and a radius of 50 $\mathrm{mm}$. One can use a linear ultrasound array, which is vertically placed and has 30 elements evenly distributed a length of 90 $\mathrm{mm}$, to horizontally scan the sample, with a step size of $3^{\circ}$ to cover the measurement area. One can also vertically scan a circular ultrasound array with a step size of $3 \mathrm{~mm}$, where the circular array may have 120 elements evenly distributed in the array. In these ways, the measurement covers 3600 detection positions, which are approximately evenly distributed in the measurement area. The sample center lies at $(0,0,0)$, the center of the measurement cylindrical surface. Fig. 4(a) shows the reconstructed $\mathrm{RF}$ absorption distribution in the $z=0 \mathrm{~mm}$

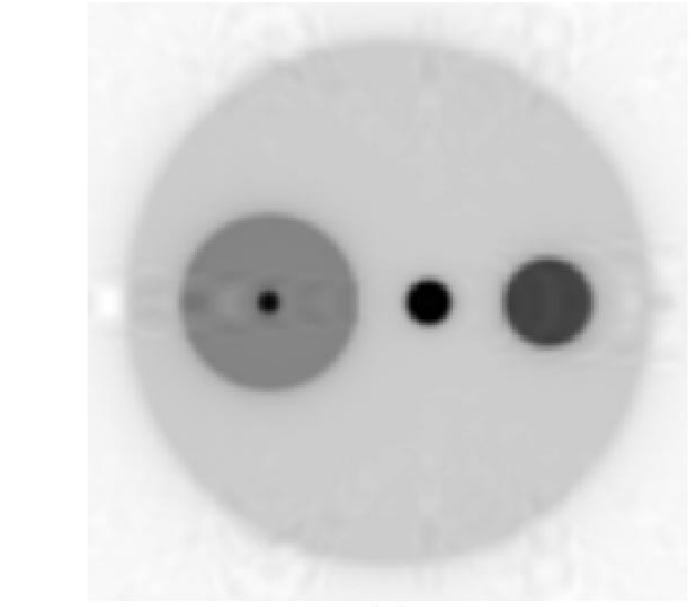

(a)

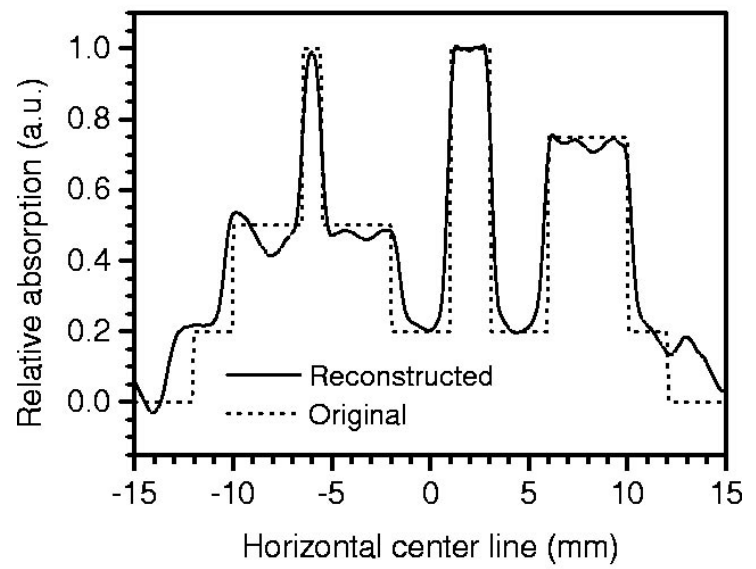

(b)

Fig. 3. Reconstructed image from planar measurement configuration using 3600 detector positions with high cutoff frequency $4 \mathrm{MHz}$. (a) Cross-sectional image at the $z=30 \mathrm{~mm}$ plane. (b) Comparison of the original and reconstructed absorption profiles along the horizontal center line.

plane and Fig. 4(b) shows the comparison of the original and reconstructed absorption profiles along the horizontal center line.

\section{Spherical Measurement Configuration}

Fig. 1(c) shows the spherical measurement configuration. To simulate a practical condition, we adopt only a half-spherical measurement area in the upper half space $(z>0)$. Suppose a quarter circular array has 30 elements and the radius of the array is $50 \mathrm{~mm}$. Then one can rotationally scan the array along its radius with a step size of $3^{\circ}$ to cover a half spherical measurement area. In this way, the, the measurement contains 3600 detection positions, which are approximately evenly distributed in the measurement area. The sample center lies $(0,0,12 \mathrm{~mm})$ inside the measurement surface. Fig. 5(a) shows the reconstructed RF absorption distribution of the $z=12 \mathrm{~mm}$ plane, and Fig. 5(b) shows the comparison of the original and reconstructed absorption profiles along the horizontal center line.

The above examples demonstrate the performance of the time-domain formulas for different measurement configurations. The reconstructed profiles are in good agreement with the original distributions. As mentioned before, with a cutoff 


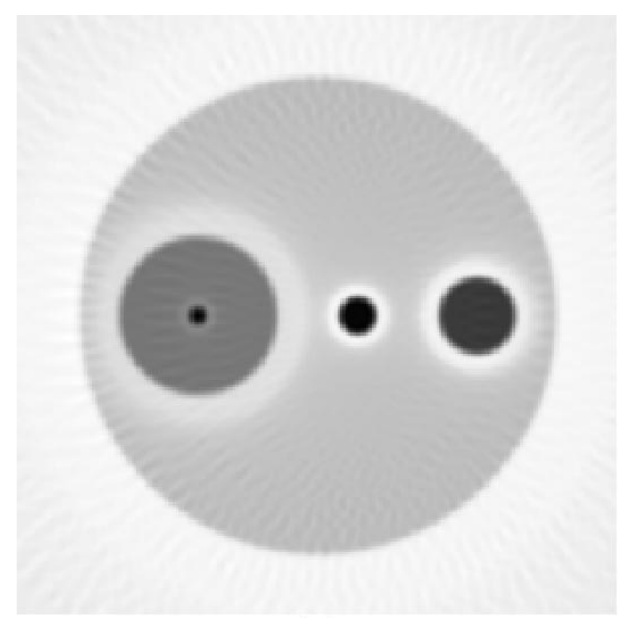

(a)

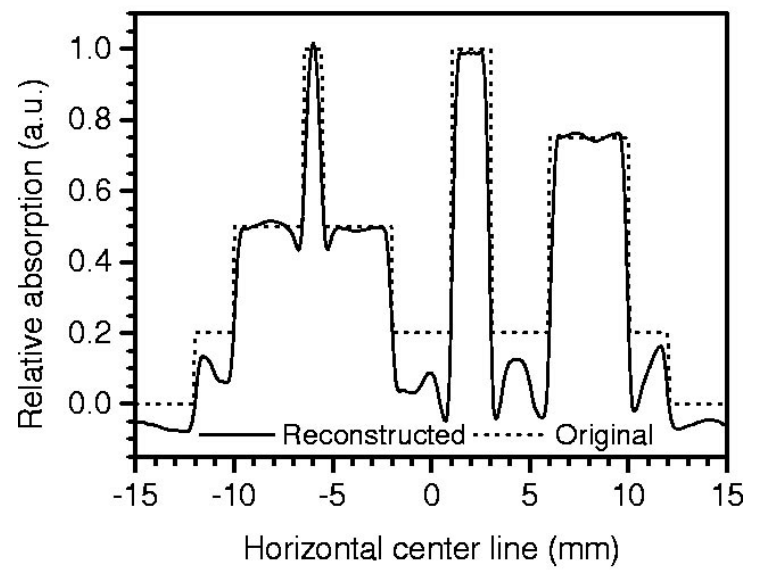

(b)

Fig. 4. Reconstructed image from cylindrical configuration using 3600 detector positions with high cutoff frequency $4 \mathrm{MHz}$. (a) Cross-sectional image at the $z=0 \mathrm{~mm}$ plane. (b) Comparison of the original and reconstructed absorption profiles along the horizontal center line.

frequency $f_{\Omega}=4 \mathrm{MHz}$, the dominative frequency in $\omega W_{\Omega}(\omega)$ is $1.7 \mathrm{MHz}$, which corresponds to an acoustic wavelength of $0.9 \mathrm{~mm}$. That explains why the small absorbers, as well as the boundaries of the big absorbers, can be faithfully reconstructed. As predicted, the flat bases of the big absorbers are not faithfully recovered, which results from the approximations of the algorithms.

However, in the absence of a high-frequency signal, the small size structure will be lost. For example, if the cutoff frequency $f_{\Omega}=1.5 \mathrm{MHz}$, the dominative frequency in $\omega W_{\Omega}(\omega)$ is about $0.6 \mathrm{MHz}$, which corresponds to an acoustic wavelength of 2.5 $\mathrm{mm}$. Without loss of generality, we will take the spherical measurement configuration as an example. The other parameters in the numerical experiment are the same as the example shown in Fig. 5. As shown in Fig. 6, not only is the small absorber nearly corrupted, but also the originally sharp borders of the big absorbers are greatly degraded.

Only a small number of detector positions affect the reconstructed images. We will again take the spherical measurement configuration as an example. Suppose a quarter circular array has only eight elements and the radius of the array is $50 \mathrm{~mm}$.

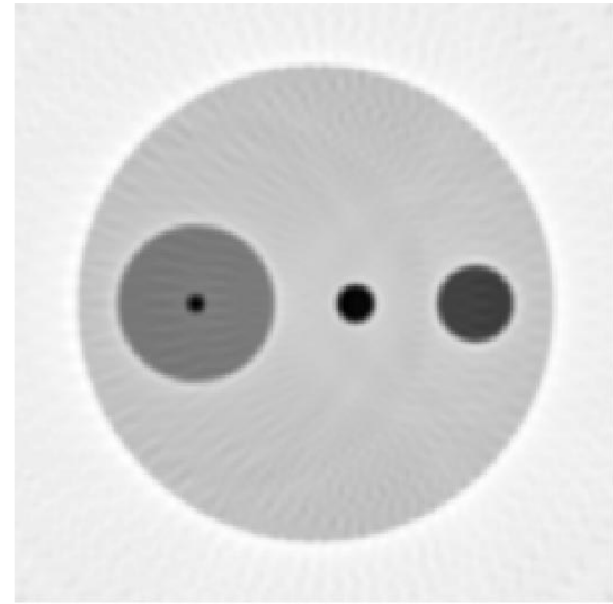

(a)

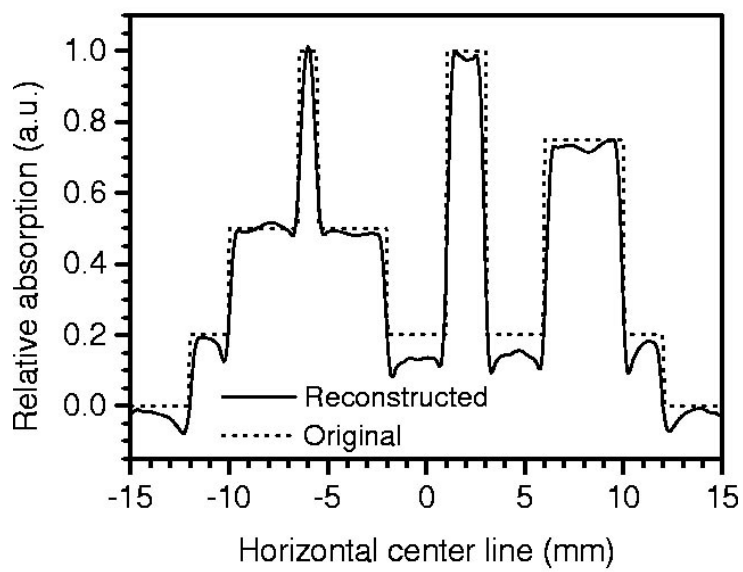

(b)

Fig. 5. Reconstructed image from spherical measurement configuration using 3600 detector positions with high cutoff frequency $4 \mathrm{MHz}$. (a) Cross-sectional image at the $z=12 \mathrm{~mm}$ plane. (b) Comparison of the original and reconstructed absorption profiles along the horizontal center line.

One must rotationally scan the array along its radius with a step size of $11.25^{\circ}$ to cover a half spherical measurement area. The other parameters in the numerical experiment are the same as in the example shown in Fig. 5. In this way, the measurement has only 256 detection positions. As shown in Fig. 7, the main structure of the sample is recovered in the reconstructed image, but a lot of noisy artifacts occur.

In addition, the signal-to-noise ratio (SNR) should be carefully considered in thermoacoustic imaging, since the amplitude of the thermoacoustic signal is small as was mentioned in Section I. In general, white noise can be suppressed by averaging over many identical data acquisitions. Denoising can also be accomplished with more elaborate methods including Fourier-based filtering and wavelet-based filtering [25]. Fortunately, reconstruction in thermoacoustic imaging is a linear addition process as shown in (16), (23), and (29). The white noise in each detector is independent of every other. If there are $n$ detectors, the SNR in the image will be improved by the square root of $n$ times through summation of the data. Of course, more detectors and more data acquisitions will increase the cost of the data acquisition time as well as the detection equipment. Actu- 


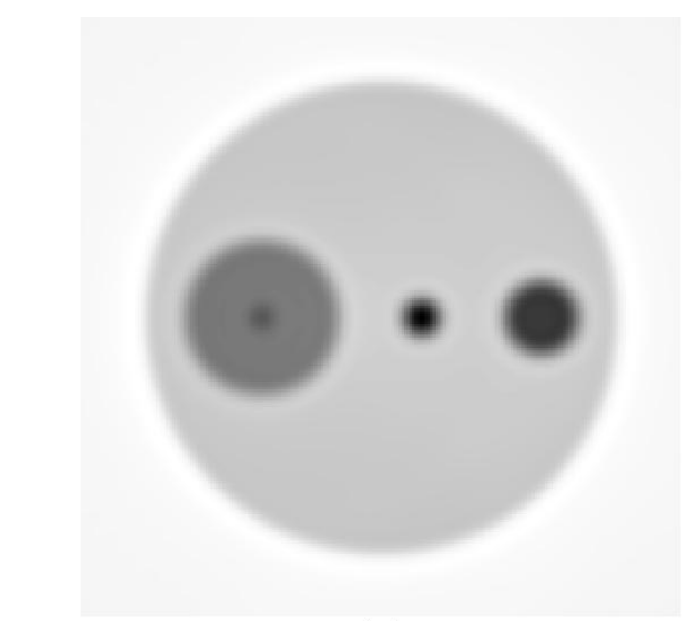

(a)

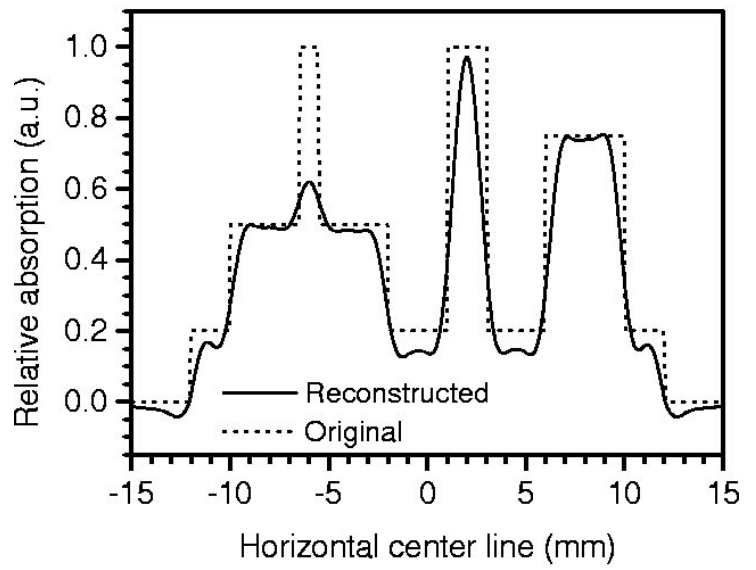

(b)

Fig. 6. Reconstructed image from spherical measurement configuration using 3600 detector positions with high cutoff frequency $1.5 \mathrm{MHz}$. (a) Cross-sectional image at the $z=12 \mathrm{~mm}$ plane. (b) Comparison of the original and reconstructed absorption profiles along the horizontal center line.

ally, as with other imaging modalities, such as magnetic resonance imaging, there is a tradeoff between SNR and the cost of data acquisition time and equipment.

In the above simulations, we consider the point-detectors. In fact, a finite detector area will limit the lateral spatial resolution and affect the axial resolution slightly [6]. A complete analytical explanation of spatial resolution related to bandwidth and detector aperture size will be reported in another paper [26].

\section{Practical ApPlications}

The time-domain reconstruction formulas-termed modified back projections - can be derived under the practical conditions discussed above. We have shown that modified back projection formulas closely approximate the rigorous formulas under the above conditions. Unlike the filtered back projection algorithm used in X-ray tomography, which uses the surface integration over intersecting planes, the modified formulas in our problems are calculated through temporal back projection and coherent summation over spherical surfaces with certain spatial weighting factors. Fortunately, due to the advantage of coherent summation, these formulas are still applicable to practical con-

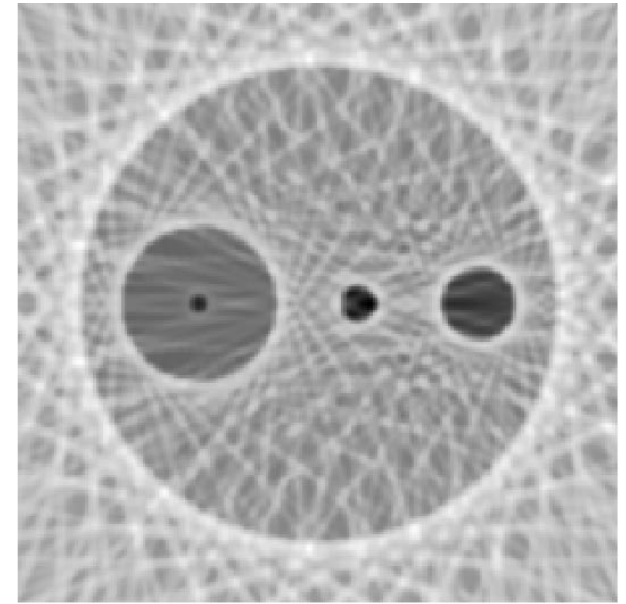

(a)

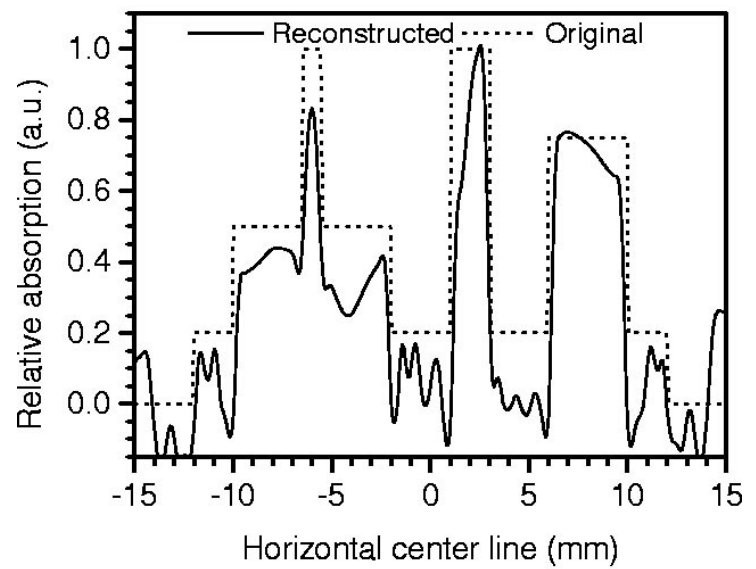

(b)

Fig. 7. Reconstructed image from spherical measurement configuration using 256 detector positions with high cutoff frequency $4 \mathrm{MHz}$. (a) Cross-sectional image at the $z=12 \mathrm{~mm}$ plane. (b) Comparison of the original and reconstructed absorption profiles along the horizontal center line.

ditions with a finite extension or partial enclosure even though they are derived from idealized recording surfaces. Of course, finite recording surfaces only provide limited spatial views, but that is adequate in practical applications.

The planar, spherical, and cylindrical recording surfaces may cover most measurement configurations. Among them the planar measurement geometry may be the easiest to implement. A two-dimensional (2-D) planar ultrasonic transducer array can be used to detect the thermoacoustic signals as in conventional ultrasound imaging. For example, Hoelen et al. [10] used this kind of recording geometry in their photoacoustic imaging. They adopted a delay-and-sum algorithm with experiential weighting factors, which worked well in dealing with their experimental data. Our research shows that the spatial weighting factor $\left[\mathbf{n}, \mathbf{n}_{0}\right]$ does exist in the back projection formula of (16) for the planar recording configuration. This is an interesting result in our theoretical analysis, which indicates that (16) should be a more accurate form than the one used by Hoelen et al..

The spherical recording configuration may be more suitable for external organ imaging such as breast cancer detection where 
in practice, only a semispherical measurement surface can be implemented. For example, Kruger et al. employed this kind of measurement geometry [7]. In their experimental system, multiple discrete transducers were mounted on a hemispherical bowl and could scan nearly a $2 \pi$ solid angle surrounding the breast volume. In the data processing, they assumed that the size of a typical absorbing object was much smaller than the detecting distance, and that the spherical surface, on which the surface integral was computed, approximated a plane. Therefore, the inverse Radon transform was approximately used to reconstruct the image as in X-ray tomography. Obviously, the above far-field condition is not strict, especially when the absorption source is far away from the center of the spherical geometry and results in reconstruction artifacts. Our theoretical analysis gives a more reasonable reconstruction formula (29), which can improve the quality of the reconstructed images.

The cylindrical recording configuration partially combines the properties of planar geometry and of spherical geometry. The reconstruction formula (23) shows a spatial weighting factor $\left[\mathbf{n}, \mathbf{n}_{0}\right] \leq 1$, which is dependent on $\left|z-z_{0}\right|$. The weighting factor reaches the maximum value $\left[\mathbf{n}, \mathbf{n}_{0}\right]=1$ at $z=z_{0}$, which indicates that the cross-sectional image of any $z_{0}$ plane is primarily determined by the data measured on the circle of the same plane. For example, if some small strong absorption sources at a size of several millimeters lie on the $z_{0}$ plane inside a weak absorption background at a size of several centimeters in diameter, a set of circular measurement data detected on a circle with a radius of several centimeters on the $z_{0}$ plane would be sufficient to yield a good cross-sectional image. In our initial work [5], [6], we used this kind of circular measurement to investigate some phantom samples and the reconstructed images agreed with the samples very well. But, if there are other absorbers outside the $z_{0}$ plane, the thermoacoustic signals from these absorbers also reach the detectors in the $z_{0}$ plane. Thus, a set of circular measurement data on the $z_{0}$ plane only could not distinguish between the absorbers on or outside of the plane. In this case, three-dimensional measurement and reconstruction must be used.

In fact, the choice of measurement configuration depends on the practical needs. From the physical point of view, these reconstruction formulas, (16), (23), and (29) for planar, cylindrical, and spherical configurations, respectively, are the same, except that the spatial weighting factors resulted from the measurement geometries. In addition, the weighting factors in the above equations are obtained through first-order approximations. In principle, high-order approximations can be derived.

Finally, it has to be pointed out that an inhomogeneous acoustic property, such as the speed of sound variation, might blur the reconstructed images. The experiments as shown in [5] and [6] demonstrated that the small speed variations between fat and muscle or gelatin did not result in significant reconstruction artifacts. The reason is that thermoacoustic waves are produced internally by RF absorption and are propagated one-way to the detectors. Thus, a small speed variation does not affect the travel time of the sound very much in a finite-length path, for example, $10 \mathrm{~cm}$, which is a typical breast diameter. Therefore, in thermoacoustic tomography, satisfactory contrast and resolution are obtainable even in tissue with a small degree of acoustic inhomogeneity.

\section{CONCLUSION}

In this paper, we have presented time-domain reconstruction algorithms for the thermoacoustic imaging of biological tissues. They are computed through temporal back projections and coherent summations over spherical surfaces with certain spatial weighting factors. Numerical experiments have demonstrated the validity of their applications. These formulas (or high-order approximations of the rigorous reconstruction formulas) can serve as the basis for time-domain thermoacoustic or photoacoustic imaging in biological tissues.

\section{APPENDIX A}

The delta function can be written in the Cartesian coordinates as

$$
\delta\left(\mathbf{r}-\mathbf{r}_{0}\right)=\delta\left(x-x_{0}\right) \delta\left(y-y_{0}\right) \delta(z)
$$

Taking Fourier transforms on both sides of (8) on variables $x$, $y$, and $z$, it can be shown that the Green's function is a triple Fourier integral of (11). If the recording surface $S_{0}$ is infinite, we may take 2-D Fourier transforms on $x_{0}$ and $y_{0}$ of $\widetilde{p}\left(x_{0}, y_{0}, k\right)$, i.e., multiplying both sides of (7) by $\exp \left(i u x_{0}+i v y_{0}\right)$ and integrating with respect to $x_{0}$ and $y_{0}$ from $-\infty$ to $+\infty$, one gets

$$
\begin{aligned}
& \int_{-\infty}^{+\infty} d x_{0} d y_{0} \exp \left(i u x_{0}+i v y_{0}\right) \widetilde{p}\left(x_{0}, y_{0}, k\right) \\
& =\frac{-i k c^{2} \eta}{2 \pi} \int_{0}^{+\infty} d z \int_{-\infty}^{+\infty} d x d y A(x, y, z) \exp (i u x+i v y) \\
& \quad \int_{-\infty}^{+\infty} d K_{z} \frac{\exp \left(-i K_{z} z\right)}{K_{z}^{2}+\rho^{2}-k^{2}}
\end{aligned}
$$

where $\rho=\sqrt{u^{2}+v^{2}},(\rho \geq 0)$.

The integral of the far right of (A2) can be computed by the contour integration $(z>0)$, because there will always be some damping of the wave in a physical system [22], [24]

$$
\begin{aligned}
& \int_{-\infty}^{+\infty} d K_{z} \frac{\exp \left(-i K_{z} z\right)}{K_{z}^{2}+\rho^{2}-k^{2}} \\
& \quad= \begin{cases}i \pi \operatorname{sgn}(k) \frac{\exp \left[i z \operatorname{sgn}(k) \sqrt{k^{2}-\rho^{2}}\right]}{\sqrt{k^{2}-\rho^{2}}}, & |k|>\rho \\
-\pi \frac{\exp \left[-z \sqrt{\rho^{2}-k^{2}}\right]}{\sqrt{\rho^{2}-k^{2}}}, & |k|<\rho\end{cases}
\end{aligned}
$$

where $\operatorname{sgn}(k)=1$ for $k \geq 0$ and $\operatorname{sgn}(k)=-1$ for $k<0$.

Here, we use the values of $k$ for $|k|>\rho$ to do the reconstruction. Those of $k$ for $|k|<\rho$ correspond to evanescent waves and will have no contribution to the reconstruction. 
In the case $|k|>\rho$ and $z>0$, (A2) becomes

$$
\begin{gathered}
\int_{-\infty}^{+\infty} \int_{0} d x_{0} d y_{0} \exp \left(i u x_{0}+i v y_{0}\right) \frac{2}{k c^{2} \eta} \widetilde{p}\left(x_{0}, y_{0}, k\right) \\
=\int_{0}^{+\infty} d z \int_{-\infty}^{+\infty} d x d y A(x, y, z) \exp (i u x+i v y) \\
\cdot \operatorname{sgn}(k) \frac{\exp \left[i z \operatorname{sgn}(k) \sqrt{k^{2}-\rho^{2}}\right]}{\sqrt{k^{2}-\rho^{2}}} .
\end{gathered}
$$

Multiplying both sides of (A4) by $\exp \left(-i u x^{\prime}-i v y^{\prime}\right)$ and integrating with respect to $u$ and $v$ letting $\rho$ from 0 to $|k|$, and further multiplying both sides of (A4) by $k \exp \left\lfloor-i z^{\prime} \operatorname{sgn}(k) \sqrt{k^{2}-\rho^{2}}\right\rfloor$ and integrating with respect to $k$ from $-\infty$ to $+\infty$, gives

$$
\begin{aligned}
& \int_{-\infty}^{+\infty} \int_{0} d x_{0} d y_{0} \int_{-\infty}^{+\infty} d k \int_{\rho=0}^{\rho=|k|} \int_{-\operatorname{loxp}} d u d v \\
& \cdot \frac{2 \widetilde{p}\left(x_{0}, y_{0}, k\right)}{k c^{2} \eta} k \exp \left[-i z^{\prime} \operatorname{sgn}(k) \sqrt{k^{2}-\rho^{2}}\right] \\
& =\int_{0}^{+\infty} d z \int_{-\infty}^{+\infty} \int_{-\infty} d x d y A(x, y, z) \\
& \quad \cdot \int_{-\infty}^{+\infty} k d k \operatorname{sgn}(k) \frac{\exp \left[i\left(z-z^{\prime}\right) \operatorname{sgn}(k) \sqrt{k^{2}-\rho^{2}}\right]}{\sqrt{k^{2}-\rho^{2}}} \\
& \left.\quad \cdot \int_{\rho=\mid}^{\rho=|k|} d u d v \exp \left[i u\left(x-x^{\prime}\right)\right] \exp \left[i v\left(y-y^{\prime}\right)\right] . \text { (A }\right)
\end{aligned}
$$

Rearranging the orders of integration of the right-hand side of (A5), we get

$$
\begin{aligned}
\text { right }= & \int_{0}^{+\infty} d z \int_{-\infty}^{+\infty} \int_{-\infty}^{+\infty} d x d y A(x, y, z) \int_{-\infty}^{+\infty} d u d v \\
& \times \exp \left[i u\left(x-x^{\prime}\right)\right] \exp \left[i v\left(y-y^{\prime}\right)\right] \\
& \cdot\left[\int_{\rho}^{+\infty} k d k \frac{\exp \left[i\left(z-z^{\prime}\right) \sqrt{k^{2}-\rho^{2}}\right]}{\sqrt{k^{2}-\rho^{2}}}\right. \\
& \left.+\int_{-\infty}^{-\rho} k d k \frac{\exp \left[-i\left(z-z^{\prime}\right) \sqrt{k^{2}-\rho^{2}}\right]}{-\sqrt{k^{2}-\rho^{2}}}\right] .
\end{aligned}
$$

If we let $w=\operatorname{sgn}(k) \sqrt{k^{2}-\rho^{2}}$, (A6) reduces to

$$
\begin{aligned}
\text { right }= & \int_{0}^{+\infty} d z \int_{-\infty}^{+\infty} \int_{-\infty}^{+\infty} d x d y A(x, y, z) \cdot \iint_{-\infty}^{+\infty} \int d u d v d w \\
& \times \exp \left[i u\left(x-x^{\prime}\right)\right] \exp \left[i v\left(y-y^{\prime}\right)\right] \exp \left[i\left(z-z^{\prime}\right) w\right] \\
= & \int_{0}^{+\infty} d z \int_{-\infty}^{+\infty} A(x, y, z) d x d y \\
& \cdot(2 \pi)^{3} \delta\left(x-x^{\prime}\right) \delta\left(y-y^{\prime}\right) \delta\left(z-z^{\prime}\right) \\
= & (2 \pi)^{3} A\left(x^{\prime}, y^{\prime}, z^{\prime}\right) .
\end{aligned}
$$

Then, substituting (A7) into (A5) and dropping the primes, we get (12) and (13).

Next, we want to show that under certain practical conditions, (12) reduces to a modified back projection formula. Replacing $K_{x}$ and $K_{y}$ in (11) with $u$ and $v$, and then taking complex conjugates of (11) and (A3), one gets

$$
\begin{aligned}
\widetilde{G}_{k}^{*}\left(\mathbf{r}, \mathbf{r}_{0}\right)= & \frac{1}{(2 \pi)^{3}} \int_{-\infty}^{+\infty} \int u d v \exp \left[i u\left(x_{0}-x\right)+i v\left(y_{0}-y\right)\right] \\
& \cdot \int_{-\infty}^{+\infty} d K_{z} \frac{\exp \left(i K_{z} z\right)}{K_{z}^{2}+\rho^{2}-k^{2}}
\end{aligned}
$$

and

$$
\begin{aligned}
& \int_{-\infty}^{+\infty} d K_{z} \frac{\exp \left(i K_{z} z\right)}{K_{z}^{2}+\rho^{2}-k^{2}} \\
& =\left\{\begin{aligned}
-i \pi \operatorname{sgn}(k) & \\
\times \frac{\exp \left[-i z \operatorname{sgn}(k) \sqrt{k^{2}-\rho^{2}}\right]}{\sqrt{k^{2}-\rho^{2}}}, & |k|>\rho, \\
-\pi \frac{\exp \left[-z \sqrt{\rho^{2}-k^{2}}\right]}{\sqrt{\rho^{2}-k^{2}}}, & |k|<\rho .
\end{aligned}\right.
\end{aligned}
$$

Then, substituting (A9) into (A8), taking the first derivative on variable $z$ of (A8) and then making a comparison with (13), one finds where

$$
\frac{\partial}{\partial z} \widetilde{G}_{k}^{*}\left(\mathbf{r}, \mathbf{r}_{0}\right)=-\frac{\pi c^{2} \eta}{2} \widetilde{K}_{k}\left(\mathbf{r}_{0}, \mathbf{r}\right)+\widetilde{\varepsilon}_{k}\left(\mathbf{r}_{0}, \mathbf{r}\right),
$$

$$
\begin{aligned}
\widetilde{\varepsilon}_{k}\left(\mathbf{r}, \mathbf{r}_{0}\right)= & \frac{1}{8 \pi^{2}} \int_{\rho=|k|}^{\rho=+\infty} d u d v \exp \left[i u\left(x_{0}-x\right)\right] \\
& \times \exp \left[i v\left(y_{0}-y\right)\right] \\
& \cdot \exp \left[-z \sqrt{\rho^{2}-k^{2}}\right]
\end{aligned}
$$

If letting $u=\rho \cos \psi, v=\rho \sin \psi, x_{0}-x=R \cos \alpha$, and $y_{0}-y=R \sin \alpha$, where $\rho=\sqrt{u^{2}+v^{2}}$ and $R=\sqrt{\left(x_{0}-x\right)^{2}+\left(y_{0}-y\right)^{2}}$, through changing the variables of integration, using the identity

$$
\frac{1}{2 \pi} \int_{0}^{2 \pi} d \phi \exp [i \rho \phi \cos (\phi-\alpha)]=J_{0}(\rho R)
$$

one can rewrite (A11) as

$$
\widetilde{\varepsilon}_{k}\left(\mathbf{r}, \mathbf{r}_{0}\right)=\frac{1}{4 \pi} \int_{|k|}^{+\infty} \rho d \rho J_{0}(\rho R) \exp \left[-z \sqrt{\rho^{2}-k^{2}}\right] .
$$

As [24] shows

$$
\begin{aligned}
\left|\widetilde{\varepsilon}_{k}\left(\mathbf{r}, \mathbf{r}_{0}\right)\right| \leq\left|\widetilde{\varepsilon}_{0}\left(\mathbf{r}, \mathbf{r}_{0}\right)\right| & =\frac{1}{4 \pi} \int_{0}^{+\infty} \rho d \rho J_{0}(\rho R) \exp (-z \rho) \\
& =\frac{z}{4 \pi\left(z^{2}+\rho^{2}\right)^{\frac{3}{2}}} \\
& =\frac{z}{4 \pi\left|\mathbf{r}-\mathbf{r}_{0}\right|^{3}}
\end{aligned}
$$


However

$$
\begin{aligned}
\frac{\partial}{\partial z} \widetilde{G}_{k}^{*}\left(\mathbf{r}, \mathbf{r}_{0}\right) & =\frac{\partial}{\partial z}\left[\frac{\exp \left(-i k\left|\mathbf{r}-\mathbf{r}_{0}\right|\right)}{4 \pi\left|\mathbf{r}-\mathbf{r}_{0}\right|}\right] \\
& =\frac{-z}{\left|\mathbf{r}-\mathbf{r}_{0}\right|}\left(\frac{1}{\left|\mathbf{r}-\mathbf{r}_{0}\right|}+i k\right) \widetilde{G}_{k}^{*}\left(\mathbf{r}, \mathbf{r}_{0}\right) .
\end{aligned}
$$

Therefore, under the condition $|k|\left|\mathbf{r}-\mathbf{r}_{0}\right| \gg 1$, one gets

$$
\left|\frac{\partial}{\partial z} \widetilde{G}_{k}^{*}\left(\mathbf{r}, \mathbf{r}_{0}\right)\right|>\frac{z|k|}{4 \pi\left|\mathbf{r}-\mathbf{r}_{0}\right|^{2}} \gg \frac{z}{4 \pi\left|\mathbf{r}-\mathbf{r}_{0}\right|^{3}} \geq\left|\widetilde{\varepsilon}_{k}\left(\mathbf{r}, \mathbf{r}_{0}\right)\right| \text {. }
$$

This means that the evanescent contribution $\widetilde{\varepsilon}$ is negligible when $|k|\left|\mathbf{r}-\mathbf{r}_{0}\right| \gg 1$ holds. Then, from (A10), we get

$$
\widetilde{K}_{k}\left(\mathbf{r}_{0}, \mathbf{r}\right) \approx-\frac{2}{\pi c^{2} \eta} \frac{\partial}{\partial z} \widetilde{G}_{k}^{*}\left(\mathbf{r}, \mathbf{r}_{0}\right) \approx \frac{i 2 k}{\pi c^{2} \eta} \frac{z}{\left|\mathbf{r}-\mathbf{r}_{0}\right|} \widetilde{G}_{k}^{*}\left(\mathbf{r}, \mathbf{r}_{0}\right) .
$$

\section{APPENDIX B}

The delta function can be expressed in the circular cylindrical coordinates [22]

$$
\begin{aligned}
\delta\left(\mathbf{r}-\mathbf{r}_{0}\right)= & \frac{1}{\rho} \delta\left(\rho-\rho_{0}\right) \delta\left(\varphi-\varphi_{0}\right) \delta\left(z-z_{0}\right) \\
= & \frac{1}{\rho} \delta\left(\rho-\rho_{0}\right) \frac{1}{2 \pi} \sum_{m=-\infty}^{+\infty} \exp \left[i m\left(\varphi-\varphi_{0}\right)\right] \\
& \cdot \frac{1}{2 \pi} \int_{-\infty}^{+\infty} \exp \left[i k_{z}\left(z-z_{0}\right)\right] d k_{z} .
\end{aligned}
$$

Assuming a similar expansion of the Green's function as

$$
\begin{aligned}
\widetilde{G}_{k}\left(\mathbf{r}, \mathbf{r}_{0}\right) & =\frac{1}{4 \pi^{2}} \sum_{m=-\infty}^{+\infty} \exp \left[i m\left(\varphi-\varphi_{0}\right)\right] \\
& \int_{-\infty}^{+\infty} d k_{z} \exp \left[i k_{z}\left(z-z_{0}\right)\right] \cdot g_{m k}\left(\rho, \rho_{0}, k_{z}\right) .
\end{aligned}
$$

Substituting (B1) and (B2) into (8), we get

$\rho^{2} \frac{d^{2} g_{m k}}{d \rho^{2}}+\rho \frac{d g_{m k}}{d \rho}+\left[\left(k^{2}-k_{z}^{2}\right) \rho^{2}-m^{2}\right] g_{m k}=-\rho \delta\left(\rho-\rho_{0}\right)$.

For the $k>0$ case, by letting $\mu=\sqrt{k^{2}-k_{z}^{2}}$, one obtains

$$
g_{m k}\left(\rho, \rho_{0}, k_{z}\right)=\frac{i \pi}{2} J_{m}(\mu \rho) H_{m}^{(1)}\left(\mu \rho_{0}\right)
$$

where if $k_{z}^{2}>k^{2}, g_{m k}\left(\rho, \rho_{0}, k_{z}\right)=I_{m}(-i \mu \rho) K_{m}\left(-i \mu \rho_{0}\right)$ with $\mu=i \sqrt{k_{z}^{2}-k^{2}}$. Therefore, (7) can be expressed in the following form:

$$
\begin{aligned}
\frac{\widetilde{p}\left(\mathbf{r}_{0}, k\right)}{-i k c^{2} \eta} & =\frac{1}{4 \pi^{2}} \iint_{V} \int d^{3} r A(\mathbf{r}) \sum_{m=-\infty}^{+\infty} \exp \left[i m\left(\varphi-\varphi_{0}\right)\right] \\
& \cdot \int_{-\infty}^{+\infty} d k_{z} \exp \left[i k_{z}\left(z-z_{0}\right)\right] g_{m k}\left(\rho, \rho_{0}, k_{z}\right) .
\end{aligned}
$$

For the idealized cylindrical recording geometry, $\widetilde{p}\left(\mathbf{r}_{0}, k\right)$ is a periodical function of angular variable $\varphi_{0}$ with a $2 \pi$ period and its extent along $z$ is infinite. Therefore, we may take a series expansion of the recorded data on variable $\varphi_{0}$ and a 1-D Fourier transform on variable $z_{0}$. Multiplying both sides of (B5) by $\exp \left(i \gamma z_{0}\right)$ and integrating with respect to $z_{0}$ from $-\infty$ to $+\infty$, and further multiplying both sides by $\exp \left(i n \varphi_{0}\right)$ and integrating with respect to $\varphi_{0}$ from 0 to $2 \pi$, one obtains

$$
\begin{aligned}
\int_{0}^{2 \pi} d \varphi_{0} & \int_{-\infty}^{+\infty} d z_{0} \frac{\widetilde{p}\left(\mathbf{r}_{0}, k\right)}{-i k c^{2} \eta} \exp \left(i n \varphi_{0}\right) \exp \left(i \gamma z_{0}\right) \\
= & \frac{1}{4 \pi^{2}} \iiint_{V} d^{3} r A(\mathbf{r}) \sum_{m=-\infty}^{+\infty} \exp (i m \varphi) \\
& \times \int_{0}^{2 \pi} d \varphi_{0} \exp \left[i(n-m) \varphi_{0}\right] \\
& \cdot \int_{-\infty}^{+\infty} d k_{z} \exp \left(i k_{z} z\right) g_{m k}\left(\rho, \rho_{0}, k_{z}\right) \\
& \times \int_{-\infty}^{+\infty} d z_{0} \exp \left[i\left(\gamma-k_{z}\right) \varphi_{0}\right] \\
= & \frac{1}{4 \pi^{2}} \iint_{V} \int d^{3} r A(\mathbf{r}) \sum_{m=-\infty}^{+\infty} \exp (i m \varphi) 2 \pi \delta_{n m} \\
& \cdot \int_{-\infty}^{+\infty} d k_{z} \exp \left(i k_{z} z\right) g_{m k}\left(\rho, \rho_{0}, k_{z}\right) 2 \pi \delta\left(\gamma-k_{z}\right) \\
= & \int_{V} \int d^{3} r A(\mathbf{r}) \exp (i n \varphi) \\
& \times \exp (i \gamma z) g_{n k}\left(\rho, \rho_{0}, \gamma\right) .
\end{aligned}
$$

Here we use the values of $k$ for $\gamma^{2}<k^{2}$ to do the reconstruction. Those values of $k$ for which $\gamma^{2}>k^{2}$ represent the evanescent waves play no role in the reconstruction. In the case of $\gamma^{2}<k^{2}$, we can rewrite (B6) as

$$
\begin{aligned}
\int_{0}^{2 \pi} d \varphi_{0} \int_{-\infty}^{+\infty} d z_{0} \frac{2}{\pi} \frac{\widetilde{p}\left(\mathbf{r}_{0}, k\right)}{k c^{2} \eta} & \\
\times \exp \left(i n \varphi_{0}\right) \exp \left(i \gamma z_{0}\right)=\iint_{V} & \int^{3} r A(\mathbf{r}) \exp (i n \varphi) \\
& \times \exp (i \gamma z) J_{n}(\mu \rho) \\
& \times H_{n}^{(1)}\left(\mu \rho_{0}\right) . \quad(\mathrm{B} 7)
\end{aligned}
$$

Multiplying both sides of (B7) by $\mu J_{n}\left(\mu \rho^{\prime}\right) / H_{n}^{(1)}\left(\mu \rho_{0}\right)$ and integrating them with respect to $\mu$ from 0 to $+\infty$, then multiplying both sides by $\exp \left(-i n \varphi^{\prime}\right)$ and summing $n$ from $-\infty$ to $+\infty$, and further multiplying both sides by $\exp \left(-i \gamma z^{\prime}\right)$ and integrating them with respect to $\gamma$ from $-\infty$ to $+\infty$, one gets

$$
\begin{gathered}
\int_{0}^{2 \pi} d \varphi_{0} \int_{-\infty}^{+\infty} d z_{0} \int_{-\infty}^{+\infty} d \gamma \exp \left[i \gamma\left(z_{0}-z^{\prime}\right)\right] \int_{0}^{+\infty} \mu d \mu \frac{2}{\pi} \frac{\widetilde{p}\left(\mathbf{r}_{0}, k\right)}{k c^{2} \eta} \\
\cdot \sum_{n=-\infty}^{+\infty} \exp \left[i n\left(\varphi_{0}-\varphi^{\prime}\right)\right] \frac{J_{n}\left(\mu \rho^{\prime}\right)}{H_{n}^{(1)}\left(\mu \rho_{0}\right)}
\end{gathered}
$$




$$
\begin{aligned}
= & \int_{-\infty}^{+\infty} d z \int_{0}^{\rho_{0}} \rho d \rho \int_{0}^{2 \pi} d \varphi A(\mathbf{r}) \int_{0}^{+\infty} d \gamma \exp \left[i \gamma\left(z-z^{\prime}\right)\right] \\
& \cdot \sum_{n=-\infty}^{+\infty} \exp \left[i n\left(\varphi-\varphi^{\prime}\right)\right] \int_{0}^{+\infty} \mu d \mu J_{n}(\mu \rho) J_{n}\left(\mu \rho^{\prime}\right) \\
= & \int_{-\infty}^{+\infty} d z \int_{0}^{\rho_{0}} \rho d \rho \int_{0}^{2 \pi} d \varphi A(\mathbf{r}) \cdot 2 \pi \delta\left(z-z^{\prime}\right) \\
& \cdot 2 \pi \delta\left(\varphi-\varphi^{\prime}\right) \cdot \frac{\delta\left(\rho-\rho^{\prime}\right)}{\rho} \\
= & (2 \pi)^{2} A\left(\rho^{\prime}, \varphi^{\prime}, z^{\prime}\right) .
\end{aligned}
$$

By dropping the primes, changing the integral variable from $\mu$ to $k$ according to $\mu=\sqrt{k^{2}-\gamma^{2}}$ and rearranging the orders of the integration, one can rewrite the (B8) as

$$
\begin{aligned}
A(\rho, \varphi, z)= & \frac{1}{2 \pi^{3}} \int_{0}^{2 \pi} d \varphi_{0} \int_{-\infty}^{+\infty} d z_{0} \int_{-\infty}^{+\infty} d \gamma \int_{+|\gamma|}^{+\infty} k d k \frac{\widetilde{p}\left(\mathbf{r}_{0}, k\right)}{k c^{2} \eta} \\
& \cdot \sum_{n=-\infty}^{+\infty} \exp \left[i n\left(\varphi_{0}-\varphi\right)\right] \\
& \times \frac{J_{n}\left(\rho \sqrt{k^{2}-\gamma^{2}}\right)}{H_{n}^{(1)}\left(\rho_{0} \sqrt{k^{2}-\gamma^{2}}\right)} \exp \left[i \gamma\left(z_{0}-z\right)\right] \\
= & \frac{1}{2 \pi^{3}} \int_{0}^{2 \pi} d \varphi_{0} \int_{-\infty}^{+\infty} d z_{0} \int_{0}^{+\infty} d k \frac{\widetilde{p}\left(\mathbf{r}_{0}, k\right)}{c^{2} \eta} \\
& \cdot \sum_{n=-\infty}^{+\infty} \exp \left[i n\left(\varphi_{0}-\varphi\right)\right] \\
& \times \int_{-k}^{+k} d \gamma \frac{J_{n}\left(\rho \sqrt{k^{2}-\gamma^{2}}\right)}{H_{n}^{(1)}\left(\rho_{0} \sqrt{k^{2}-\gamma^{2}}\right)} \exp \left[i \gamma\left(z_{0}-z\right)\right] .
\end{aligned}
$$

Equation (B9) can be easily written in the forms of (18) and (19).

Next, we want to show that (18) can be reduced to a modified back projection under certain conditions. When $\xi \gg 1$, according to the asymptotic expansions of the Hankel function, we get

$$
H_{n}^{(1)}(\xi) H_{n}^{(2)}(\xi) \approx \frac{2}{\pi \xi} .
$$

Assuming $\rho_{0} \sqrt{k^{2}-\gamma^{2}} \gg 1$, i.e., $\rho_{0} k \gg 1$, one can approximate

$\frac{1}{H_{n}^{(1)}\left(\rho_{0} \sqrt{k^{2}-\gamma^{2}}\right)} \approx \frac{\pi}{2} \rho_{0} \sqrt{k^{2}-\gamma^{2}} H_{n}^{(2)}\left(\rho_{0} \sqrt{k^{2}-\gamma^{2}}\right)$.

Therefore

$$
\widetilde{K}_{r}\left(\mathbf{r}, \mathbf{r}_{0}\right) \approx \frac{1}{4 \pi^{2} c^{2} \eta} \sum_{n=-\infty}^{+\infty} \exp \left[i n\left(\varphi_{0}-\varphi\right)\right]
$$

$$
\begin{aligned}
& \times \int_{-k}^{+k} d \gamma \exp \left[i \gamma\left(z_{0}-z\right)\right] \\
& \cdot \sqrt{k^{2}-\gamma^{2}} J_{n}\left(\rho \sqrt{k^{2}-\gamma^{2}}\right) \\
& \times H_{n}^{(2)}\left(\rho_{0} \sqrt{k^{2}-\gamma^{2}}\right) .
\end{aligned}
$$

We can argue that the values of $\gamma$ for $\gamma^{2}>k^{2}$ do not contribute to the reconstruction. Taking the complex conjugate of the Green's function in (B2) and replacing $k_{z}$ by $\gamma$, we may exclude these $\gamma$ satisfying $\gamma^{2}>k^{2}$ and approximate the Green's function as

$$
\begin{gathered}
\widetilde{G}_{k}^{*}\left(\mathbf{r}, \mathbf{r}_{0}\right) \approx-\frac{i}{8 \pi} \sum_{n=-\infty}^{+\infty} \exp \left[i n\left(\varphi_{0}-\varphi\right)\right] \int_{-k}^{+k} d \gamma \exp \left[i \gamma\left(z_{0}-z\right)\right] \\
\cdot J_{n}\left(\rho \sqrt{k^{2}-\gamma^{2}}\right) H_{n}^{(2)}\left(\rho \sqrt{k^{2}-\gamma^{2}}\right) .
\end{gathered}
$$

Letting $z_{1}=z_{0}-z$, the second-order partial derivative of (B13) with respect to $z_{1}$ has the following relation:

$$
\frac{\partial^{2}}{\partial z_{1}^{2}} \sim-\gamma^{2}
$$

Comparing (B12) with (B13), we get

$$
\widetilde{K}_{k}\left(\mathbf{r}_{0}, \mathbf{r}\right)=\frac{i 2 k}{\pi c^{2} \eta} \sqrt{1+\frac{1}{k^{2}} \frac{\partial^{2}}{\partial z_{1}^{2}}} \widetilde{G}_{k}^{*}\left(\mathbf{r}, \mathbf{r}_{0}\right) .
$$

Under the condition $k\left|\mathbf{r}-\mathbf{r}_{0}\right| \gg 1$

$$
\begin{aligned}
\frac{1}{k^{2}} \frac{\partial^{2}}{\partial z_{1}^{2}} \widetilde{G}_{k}^{*}\left(\mathbf{r}, \mathbf{r}_{0}\right) & =\frac{1}{k^{2}} \frac{\partial^{2}}{\partial z_{1}^{2}}\left[\frac{\exp \left(-i k\left|\mathbf{r}-\mathbf{r}_{0}\right|\right)}{4 \pi\left|\mathbf{r}-\mathbf{r}_{0}\right|}\right] \\
& \approx-\frac{\left(z_{0}-z\right)^{2}}{\left|\mathbf{r}-\mathbf{r}_{0}\right|^{2}} \widetilde{G}_{k}^{*}\left(\mathbf{r}, \mathbf{r}_{0}\right) .
\end{aligned}
$$

Then, (B15) approximates to

$$
\widetilde{K}_{k}\left(\mathbf{r}, \mathbf{r}_{0}\right) \approx \frac{i 2 k}{\pi c^{2} \eta} \sqrt{1-\frac{\left(z_{0}-z\right)^{2}}{\left|\mathbf{r}-\mathbf{r}_{0}\right|^{2}}} \widetilde{G}_{k}^{*}\left(\mathbf{r}, \mathbf{r}_{0}\right)
$$

\section{REFERENCES}

[1] R. A. Kruger, K. K. Kopecky, A. M. Aisen, D. R. Reinecke, G. A. Kruger, and W. L. Kiser, "Thermoacoustic CT with radio waves: A medical imaging paradigm," Radiology, vol. 211, pp. 275-278, 1999.

[2] G. Ku and L.-H. V. Wang, "Scanning thermoacoustic tomography in biological tissues," Med. Phys., vol. 27, pp. 1195-1202, 2000.

[3] — - "Scanning microwave-induced thermoacoustic tomography: Signal, resolution, and contrast," Med. Phys., vol. 28, pp. 4-10, 2001.

[4] M.-H. Xu, G. Ku, and L.-H. V. Wang, "Microwave-induced thermoacoustic tomography using multi-sector scanning," Med. Phys., vol. 28, pp. 1958-1963, 2001.

[5] M.-H. Xu and L.-H. V. Wang, "Time-domain reconstruction for thermoacoustic tomography in a spherical geometry," IEEE Med. Imag., vol. 21, pp. 814-822, July 2002.

[6] - "Pulsed-microwave-induced thermoacoustic tomography: Filtered backprojection in a circular measurement configuration," Med. Phys., vol. 29, pp. 1661-1669, 2002.

[7] R. A. Kruger, D. R. Reinecke, and G. A. Kruger, "Thermoacoustic computed tomography-technical considerations," Med. Phys., vol. 26, pp. 1832-1837, 1999.

[8] R. A. Kruger, K. D. Miller, H. E. Reynolds, W. L. Kiser, Jr, D. R. Reinecke, and G. A. Kruger, "Breast cancer in vivo: Contrast enhancement with thermoacoustic CT at $434 \mathrm{MHz}$-feasibility study," Radiology, vol. 216, pp. 279-283, 2000. 
[9] P. Y. Liu, "The P-transform and photoacoustic image reconstruction," Phys. Med. Biol., vol. 43, pp. 667-674, 1998.

[10] C. G. A. Hoelen and F. F. M. de Mul, "Image reconstruction for photoacoustic scanning of tissue structures," Appl. Opt., vol. 39, no. 31, pp. 5872-5883, Nov. 2000.

[11] R. O. Esenaliev, A. A. Karabutov, and A. A. Oraevsky, "Sensitivity of laser opto-acoustic imaging in detection of small deeply embedded tumors," IEEE J. Sel. Top. Quantum Electron., vol. 5, pp. 981-988, July-Aug. 1999.

[12] K. P. Kostli, M. Frenz, H. P. Weber, G. Paltauf, and H. Schmidt-Kloiber "Optoacoustic infrared spectroscopy of soft tissue," J. Appl. Phys., vol. 88, pp. $1632-1637,2000$.

[13] C. C. Johnson and A. W. Guy, "Nonionizing elecromagnetic wave effects in biological materials and systems," Proc. IEEE, vol. 60, pp. 692-718, 1972.

[14] F. A. Duck, Physical Properties of Tissue. San Diego, CA: Academic, 1990.

[15] V. E. Gusev and A. A. Karabutov, Laser Optoacoustics. New York: American Institute of Physics, 1993.

[16] P. N. T. Wells, "Ultrasonic imagining of the human body," Rep. Prog. Phy., vol. 62, pp. 671-722, 1999.

[17] L. V. Zhigilei and B. J. Garrison, "Microscopic mechanisms of laser ablation of organic solids in the thermal and stress confinement irradiation regimes," J. Appl. Phys., vol. 88, pp. 1281-1298, 2000.

[18] Y. V. Zhulina, "Optimal statistical approach to optoacoustic image reconstruction," Appl. Opt., vol. 39, no. 32, pp. 5971-5977, Nov. 2000

[19] K. P. Kostli, M. Frenz, H. Bebie, and H. P. Weber, "Temporal backward projection of optoacoustic pressure transients using Fourier transform methods," Phys. Med. Biol., vol. 46, pp. 1863-1872, 2001

[20] Y. Xu, D. Feng, and L.-H. V. Wang, "Exact frequency-domain reconstruction for thermoacoustic tomography-I: Planar geometry," IEEE Med. Imag., vol. 21, pp. 823-828, July 2002.

[21] Y. Xu, M.-H. Xu, and L.-H. V. Wang, "Exact frequency-domain reconstruction for thermoacoustic tomography-II: Cylindrical geometry," IEEE Med. Imag., vol. 21, pp. 829-833, July 2002.

[22] G. B. Arfken and H. J. Weber, Mathematical Methods for Physicists. San Diego, CA: Academic, 1995

[23] G. J. Diebold, T. Sun, and M. I. Khan, "Photoacoustic monopole radiation in one, two, and three dimensions," Phys. Rev. Lett., vol. 67, pp. 3384-3387, 1991.

[24] S. J. Norton and M. Linzer, "Ultrasonic reflectivity imaging in three dimensions: Exact inverse scattering solutions for plane, cylindrical, and spherical apertures," IEEE Trans. Biomed. Eng., vol. BME-28, pp. 202-220, 1981

[25] The Image Processing Handbook, CRC, Boca Raton, FL, 1992.

[26] M.-H. Xu and L.-H. V. Wang, "Analytic explanation of spatial resolution related to bandwidth and detector aperture size in thermoacoustic or photoacoustic reconstruction," Phys. Rev. E, vol. 67, no. 056605, 2003.
Minghua Xu received the B.S. degree in physics from Qingdao University of Oceanography, Qingdao, China, in 1994, and the M.S. and Ph.D. degrees in acoustics from Nanjing University, Jiangsu, China, in 1996 and 1999, respectively. He is working towards the Ph.D. degree in biomedical engineering at Texas A\&M University, College Station.

His main research topic is biomedical imaging.

Yuan Xu received the B.S. degree in physics from Wuhan University, Wuhan, P. R. China, in 1992, the M.S. degree in physics from Shanghai Institute of Optics and Fine Mechanics, Shanghai, P. R. China, in 1995, and the Ph.D. degree in physics from Institute of Physics, Chinese Academy of Sciences, Beijing, P. R. China, in 1999. He is now a graduate student with the department of Biomedical Engineering in Texas A\&M University, College Station.

His special fields of interest are thermoacoustic tomography and ultrasound tomography, and their applications to medical imaging.

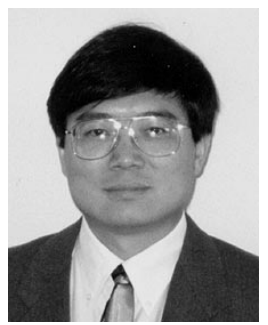

Lihong V. Wang (M'96-SM'00) received the Ph.D. degree in electrical engineering from Rice University, Houston, TX, in 1992.

He worked for University of Texas M.D Anderson Cancer Center, a top-ranked cancer institution, as an Assistant Professor. He was promoted to Professor of Biomedical Engineering and Electrical Engineering at Texas A\&M University, College Station, in 2002. His research focus is on nonionizing biophotonic imaging for early cancer detection. His group pioneered acousto-optical tomography, spectroscopic oblique-incidence reflectometry, thermo-acoustic tomography, photo-acoustic tomography, and Mueller-matrix optical coherence tomography. His program for Monte Carlo modeling of photon transport in biological tissues has been used worldwide (available at http://oilab.tamu.edu).

Dr. Wang is a fellow of American Institute for Medical and Biological Engineering, and a member of American Physical Society, Biomedical Engineering Society, Engineering in Medicine and Biology, Society Optical Society of America, and Society of Photo-Optical Instrumentation Engineers. He received the NIH FIRST Award, NSF CAREER Award, Johnson \& Johnson Outstanding Young Scientist Award, Texas A\&M TEES Select Young Faculty Award, Texas A\&M TEES Faculty Fellow Award (twice), Texas A\&M Ernest A. Baetz Faculty Fellow Award, Texas A\&M TEES Senior Faculty Fellow Award, and Texas A\&M University Faculty Fellow. He is listed in Who's Who in Science and Engineering. He has been an associate editor for the Annals of Biomedical Engineering, Journal of Biomedical Optics, and Applied Optics. 